Universidade de São Paulo

Faculdade de Medicina de Ribeirão Preto

\section{0}

Confiabilidade, reprodutibilidade e eficiência do enfaixamento compressivo funcional, no tratamento do linfedema secundário ao tratamento do câncer de mama
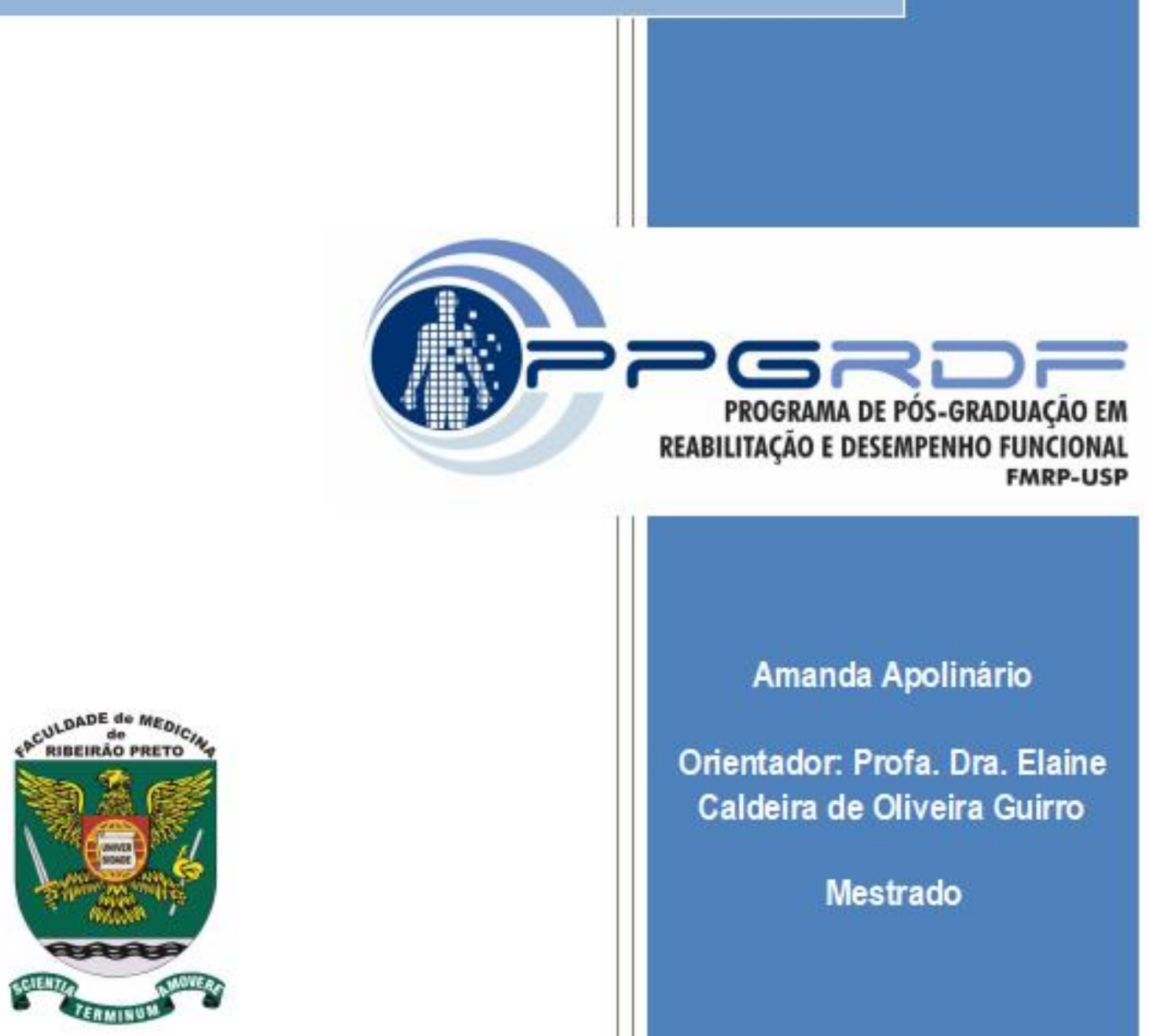

\title{
Amanda Apolinário
}

Orientador: Profa. Dra. Elaine Caldeira de Oliveira Guirro

\section{Mestrado}


FACULDADE DE MEDICINA DE RIBEIRÃO PRETO

Amanda Apolinário

Confiabilidade, reprodutibilidade e eficiência do enfaixamento compressivo funcional, no tratamento do linfedema secundário ao tratamento do câncer de mama

Ribeirão Preto 
Amanda Apolinário

Confiabilidade, reprodutibilidade e eficiência do enfaixamento
compressivo funcional, no tratamento do linfedema secundário ao
tratamento do câncer de mama

Dissertação apresentada ao Programa de PósGraduação em Reabilitação e Desempenho Funcional - FMRP/USP, pelo Departamento de Ciências da Saúde para obtenção do Título de Mestre em Ciências.

Área de concentração: Fisioterapia

Orientadora: Profa. Dra. Elaine Caldeira de Oliveira Guirro

Versão corrigida. A versão original encontra-se disponível tanto na Biblioteca da Unidade que aloja o Programa, quanto na Biblioteca Digital de Teses e Dissertações da USP (BDTD).

\section{Ribeirão Preto}




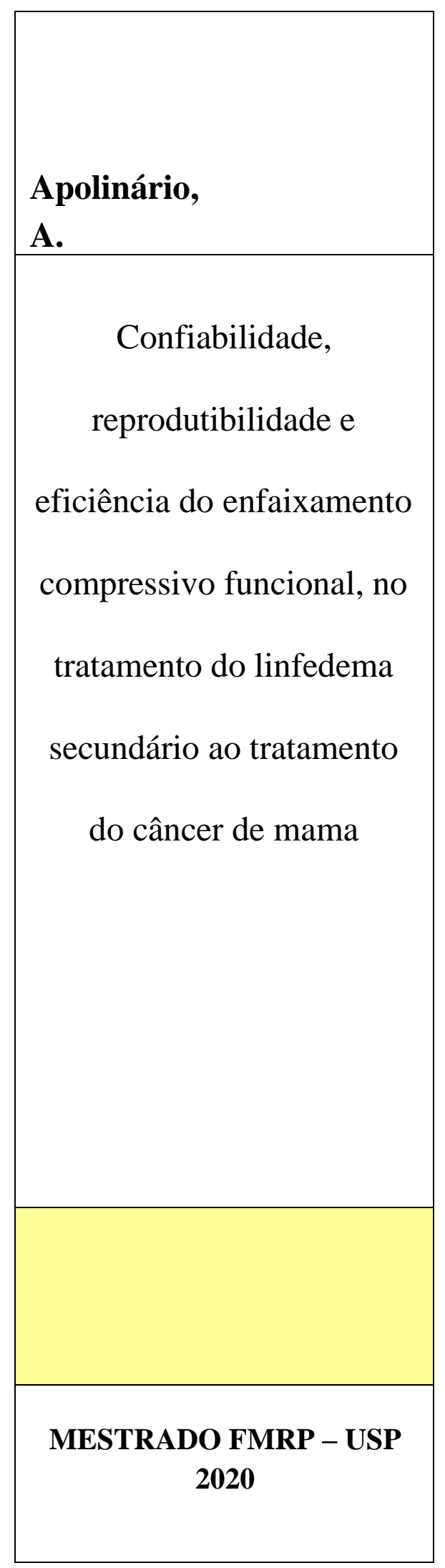




\title{
AUTORIZO A REPRODUÇÃO E DIVULGAÇÃO TOTAL OU PARCIAL DESTE TRABALHO, POR QUALQUER MEIO CONVENCIONAL OU ELETRÔNICO, PARA FINS DE ESTUDO E PESQUISA, DESDE QUE CITADA A FONTE.
}

\author{
Catalogação da Publicação
}

Serviço de Documentação da Faculdade de Medicina

Faculdade de Medicina de Ribeirão Preto da Universidade de São Paulo

Apolinário, Amanda

Confiabilidade, reprodutibilidade e eficiência do enfaixamento compressivo funcional, no tratamento do linfedema secundário ao tratamento do câncer de mama, 2020.

63 f.: il.; $30 \mathrm{~cm}$

Orientadora: Guirro, Elaine Caldeira de Oliveira.

Dissertação (Mestrado) - Programa de Pós-Graduação em Reabilitação e Desempenho Ciências da Saúde de Ribeirão Preto, Universidade de São Paulo - USP.

1. Câncer de mama. 2. Fisioterapia. 4. Linfedema. 
Nome: Amanda Apolinário

Título: Confiabilidade, reprodutibilidade e eficiência do enfaixamento compressivo

funcional, no tratamento do linfedema secundário ao tratamento do câncer de mama.

Aprovado em:

Dissertação apresentada ao Programa de PósGraduação em Reabilitação e Desempenho Funcional - FMRP/USP, para obtenção do Título de Mestre em Ciências.

Área de Concentração: Fisioterapia

Banca Examinadora

Prof. Dr. Instituição:

Julgamento: Assinatura:

Prof. Dr. Instituição:

Julgamento: Assinatura:

Prof. Dr. Instituição: Assinatura: 


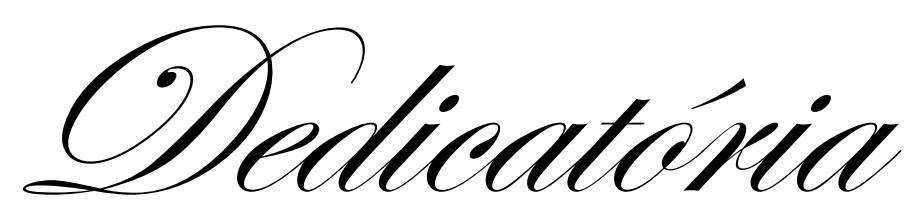


Dedica este traballho a minha famelia, Gaslos, OMarrinilua, OSnderson. o. Clliguel. 


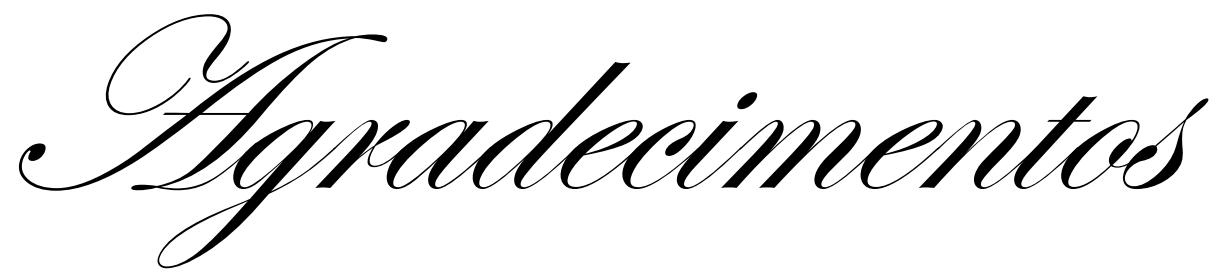




\section{AGRADECIMENTOS}

É com muito amor e carinho que venho agradecer a Deus e as pessoas que estiveram comigo durante essa fase de conclusão de mestrado. Agradeço primeiramente a Deus, por seu cuidado, que ao longo dessa caminhada sempre me deu forças para superar cada batalha, e, sem ele, nada disso seria possível. Não posso deixar de agradecer também a cada pessoa que ele colocou em meu caminho para tornar meus dias mais alegres e leves.

Agradeço carinhosamente a meus pais, Carlos e Marinilva, e a meu irmão Anderson, que em cada desafio me deram todo apoio e suporte para estar aqui, concluindo mais uma etapa. À todos, deixo a minha eterna gratidão por toda ajuda, seja ela emocional, companherismo ou financeira. Eu dedico esse trabalho a vocês, às pessoas que mais amo.

Hoje posso garantir com todo zelo que vocês são a base de todas as minhas conquistas, e nada seria possível sem vocês. E mais uma vez, agradeço a Deus pelo seu maravilhoso cuidado quando colocou vocês para serem minha base. Agradeço também ao meu companheiro Miguel, que fez parte dessa etapa, sempre disposto a ouvir, a entender e por ajudar-me em todos os momentos.

Não obstante, deixo também a minha gratidão e admiração pela Professora Elaine, que me recebeu mais uma vez em seu laboratório de abraços abertos, porém com novos desafios, não como uma aluna de inciação científica, e sim como uma aluna de mestrado. Agradeço por todo os ensinamentos, paciência e aprendizado que pude adquirir durante esses anos. Agradeço também a toda equipe do REMA e cada paciente que me acolheu com tanto carinho.

Agradeço às minhas colegas de laboratório Monique, Cristina, Ana Paula, Carla e Daniela pela imensa ajuda e companherismo, sem vocês também não seria possível completar essa etapa. Aos alunos de inciação científica, em especial Érica, que nós ajudamos e aprendemos juntas, deixo meu sentimento de gratidão pela amizade e todo carinho durante esse tempo, e aos alunos Giovani, Aline e Guilherme, que também não mediram esforços para ajudar durante as coletas. 
Agradeço à Coordenação de Aperfeiçoamento de Pessoal de Nível Superior - Brasil (CAPES) pelo apoio financeiro - Código de Financiamento 001. 


\section{RESUMO}

Apolinário, A. Confiabilidade, reprodutibilidade e eficiência do enfaixamento compressivo funcional, no tratamento do linfedema secundário ao tratamento do câncer de mama. 2020. 63 f. Dissertação (Mestrado) - Faculdade de Medicina de Ribeirão Preto, Universidade de São Paulo, Ribeirão Preto, 2020

Mulheres submetidas ao tratamento do câncer de mama com linfedema de membro superior foram avaliadas em dois estudos. $\mathrm{O}$ objetivo do primeiro estudo foi avaliar a confiabilidade, reprodutibilidade do enfaixamento compressivo funcional (ECF). O segundo estudo teve como objetivo avaliar a eficiência na circulação sanguínea, na funcionalidade e na pressão exercida em diferentes técnicas de enfaixamento compressivo funcional (ECF). O primeiro estudo avaliou a confiabilidade interexaminadores e intraexaminadores da técnica, aplicada por dois avaliadores independentes e previamente treinados, com período wash out de sete dias. Incluiu 45 mulheres com média de idade 64.88(DP=10.01) anos. O coeficiente de correlação intraclasse $\left(\mathrm{ICC}_{2,1}\right)$ foi empregado para determinar a reprodutibilidade e confiabilidade interexaminador e intraexaminador, com intervalo de confiança a 95\%. O segundo estudo visou verificar a eficiência de diferentes técnicas de ECF na funcionalidade, pressão exercida, e circulação do membro superior afetado por linfedema com delineamento aleatório cross over e período wash out de sete dias. Para tanto, foram avaliadas 23 mulheres com média de idade 64.54(DP=11.89) anos, sendo eficiência do ECF testada pela aplicação aleatória de duas diferentes técnicas (espiral e oito), em relação a pressão aplicada em $\mathrm{mmHg}$ no repouso, e durante o movimento. A funcionalidade do membro superior foi avaliada por meio do teste de Jebsen Taylor, aplicado antes e com ECF em ambos os membros. A avaliação da influência do enfaixamento na circulação foi determinada por meio do ultrassom Doppler, antes e após a aplicação do teste. A reprodutibilidade apresentou confiabilidade interexaminador na região de braço foi considerada baixa, e na região de antebraço de baixa a moderada. A confiabilidade intraexaminador foi considerada de baixa a alta na região de braço e antebraço. $\mathrm{O}$ segundo estudo apresentou resultado significativo no aumento da velocidade e pico do fluxo arterial e venoso após ECF, tanto na técnica em oito e em espiral $(\mathrm{p}=0.0001)$, quando comparado ao repouso. A técnica em oito apresentou uma maior pressão exercida sobre o tecido quando comparada a espiral ( $\mathrm{p}=$ 0.0001) durante o movimento, e houve diferença significativamente maior no tempo de execução do teste de função manual Jebsen Taylor quando realizado com ECF comparado a sem ECF. Diante dos resultados obtidos, pode-se concluir que o ECF em espiral apresenta confiabilidade baixa a moderada interexaminador e intraexaminador. A técnica em oito apresentou maior pressão em movimento quando comparado a técnica em espiral. Ambas as técnicas de ECF promoveram incremento na circulação sanguínea, e prejuízo funcional do membro envolvido.

Palavras-chave: Câncer de mama; Linfedema; Fisioterapia. 


\begin{abstract}
Apolinário, A. Reliability, reproducibility and efficiency of functional compressive bandaging in the treatment of lymphedema secondary to the treatment of breast cancer. $2020.63 \mathrm{f}$. Dissertation (Master) - Medicine School of Ribeirão Preto, University of São Paulo, Ribeirão Preto, 2020.
\end{abstract}

Women undergoing treatment of breast cancer with lymphedema of the upper limb were evaluated in two studies. The objective of the first study was to assess the reliability, reproducibility of the functional compressive bandage (ECF). The second study aimed to evaluate the efficiency of blood circulation, functionality and pressure exerted in different techniques of functional compressive bandaging (ECF). The first study evaluated the intraexaminer and inter-examiner reliability of the technique, applied by two independent and previously trained evaluators, with a wash out period of seven days. It included 45 women with a mean age of $64.88(\mathrm{SD}=10.01)$ years. The intraclass correlation coefficient (ICC2.1) was used to determine intra-examiner and inter-examiner reproducibility and reliability, with a $95 \%$ confidence interval. The second study aimed to verify the efficiency of different ECF techniques on functionality, pressure exerted, and circulation of the upper limb affected by lymphedema with a random cross over design and wash out period of seven days. For this purpose, 23 women with a mean age of $64.54(\mathrm{SD}=11.89)$ years were evaluated, and the efficiency of the ECF was tested by the random application of two different techniques (spiral and eight), in relation to the pressure applied in $\mathrm{mmHg}$ at rest, and during the movement. Upper limb functionality was assessed using the Jebsen Taylor test, applied before and with ECF on both limbs. The assessment of the influence of the bandage on the circulation was determined by means of Doppler ultrasound, before and after the application of the test. Reproducibility showed intra-examiner reliability in the arm region was considered low, and in the forearm region low to moderate. Inter-rater reliability was considered low to high in the arm and forearm region. The second study showed a significant result with an increase in the velocity and peak of arterial and venous after ECF both in the technique in eight and in a spiral $(p=0.0001)$ when compared to rest. The technique in eight showed a greater pressure exerted on the tissue when compared to the spiral $(\mathrm{p}=0.0001)$ during the movement, and there was a significantly greater difference in the execution time of the Jebsen Taylor manual function test when performed with ECF compared to without ECF. In view of the results obtained, it can be concluded that the spiral ECF has low to moderate intra-examiner and inter-examiner reliability. The technique in eight showed greater pressure in movement when compared to the spiral technique. Both ECF techniques promoted an increase in blood circulation, and functional impairment of the involved limb.

Keywords: Lymphedema; Breast cancer; Physical Therapy 


\section{LISTA DE FIGURAS}

Figura 1 Imagem ilustrativa da mensuração da perimetria.

Figura 2 Equipamento para mensuração da pressão, composto por uma bolsa inflável 29 de borracha conectada a um manômetro (A). Posicionamento do equipamento após enfaixamento (B).

Figura 3 Fluxograma do primeiro estudo da confiabilidade do enfaixamento compressivo funcional.

Figura 4 Fluxograma do segundo estudo da eficiência do enfaixamento compressivo funcional.

Figura 5 Itens do teste de funcionalidade Jebsen Taylor, composto por diferentes objetos.

Figura 6 Equipamento de ultrassom Doppler SONARA/Tek (A). Avaliação da circulação sanguínea (B). 


\section{LISTA DE TABELAS}

Tabela 1 Características gerais das mulheres do primeiro do estudo da confiabilidade 25 do enfaixamento compressivo funcional.

Tabela 2 Características gerais das mulheres do segundo estudo da eficiência do 26 enfaixamento compressivo funcional.

Tabela 3 Resultados da confiabilidade intraexaminador da técnica de enfaixamento 36 compressivo funcional, modalidade em espiral.

Tabela $4 \begin{aligned} & \text { Resultados da confiabilidade interexaminador } \\ & \text { compressivo funcional, modalidade em espiral. }\end{aligned}$

Tabela 5 Média (desvio padrão) das variáveis avaliadas e a comparação entre as situações no ultrassom Doppler $(\mathrm{cm} / \mathrm{s})$ analisadas, velocidade média do fluxo arterial e venoso, e pico do fluxo arterial e venoso do vaso braquial, no membro superior afetado, em repouso e após o teste de função manual sem e com enfaixamento compressivo funcional (espiral e em oito).

Tabela 6 Resultados da comparação das variáveis do ultrassom Doppler $(\mathrm{cm} / \mathrm{s})$ analisadas, velocidade média do fluxo arterial e venoso, e pico do fluxo arterial e venoso do vaso braquial, no membro superior afetado, após o teste de função manual com enfaixamento compressivo funcional (espiral e em oito).

Tabela 7 Média (desvio padrão) das variáveis avaliadas e a comparação da pressão $(\mathrm{mmHg})$ exercida pelo enfaixamento compressivo funcional em espiral ou em oito sobre o tecido, durante o repouso e o momento, na região de braço e antebraço.

Tabela 8 Média (desvio padrão) e a comparação entre as variáveis de Jebsen Taylor sem ou com enfaixamento compressivo funcional em espiral ou em oito no membro superior.

Tabela 9 Resultados da comparação entre os tipos de enfaixamanto compressivo funcional e as variáveis de Jebsen Taylor com enfaixamento compressivo funcional. 


\section{SUMÁRIO}

1. INTRODUÇÃ

2. OBJETIVO …................................................................................................................ 22

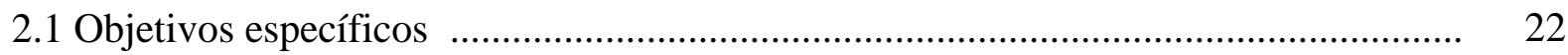

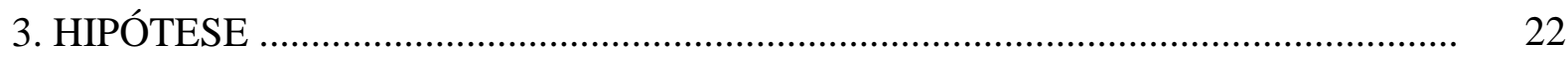

4. MATERIAL E MÉTODOS ........................................................................... 24

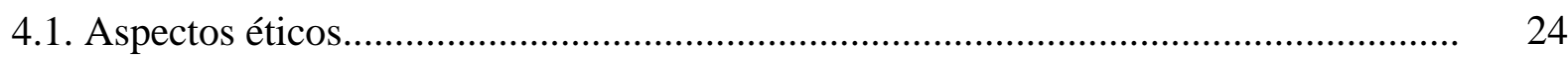

4.2. Tipos de pesquisa e delineamento do estudo ........................................................ 24

4.3. Amostra ........................................................................................... 25

4.4. Critérios de inclusão .................................................................................... 26

4.5. Critérios de exclusão ....................................................................................... 26

4.6. Avaliação inicial .................................................................................... 27

4.7. Primeiro estudo - Confiabilidade do enfaixamento compressivo funcional ............. 28

4.8. Segundo estudo - Eficiência das diferentes técnicas do enfaixamento compressivo 30 funcional

4.8.1. Enfaixamento compressivo funcional (ECF) .................................................... 31

4.8.2. Avaliação do fluxo sanguíneo............................................................................. 32

4.8.3. Teste de função manual Jebson Taylor ................................................................... 33

4.9. Análise estatística ............................................................................................. 34

5. RESULTADOS …...................................................................................................... 36

5.1. Resultados referentes à confiabilidade do enfaixamento compressivo funcional 36

5.2. Comparações do comportamento sanguíneo sem e com enfaixamento compressivo 37 funcional

5.3. Comparação da pressão exercida pelo enfaixamento compressivo funcional nas 40 diferentes técnicas (espiral e oito)

5.4. Comparação do teste de funcionalidade de Jebsen Taylor com e sem enfaixamento 42 compressivo funcional.

6. DISCUSSÃ 0 ..................................................................................................................... 46

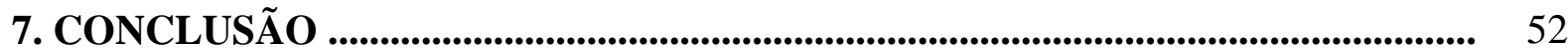

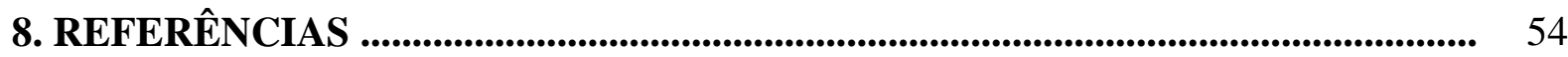

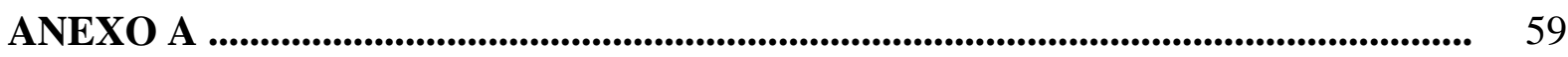

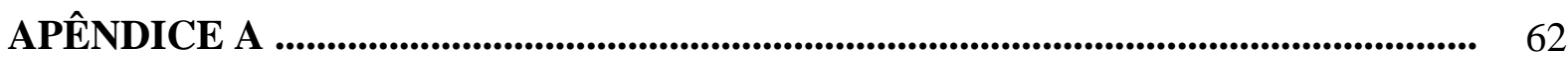


- Pretredueña 


\section{INTRODUÇÃO}

O procedimento utilizado prioritariamente para tratar o câncer de mama atualmente é o chamado processo cirúrgico, em suas mais variadas formas, conforme o estágio da doença, e associado à biópsia do linfonodo sentinela, linfadenectomia axilar, obesidade, tratamentos adjuvantes como a quimioterapia e radioterapia, bem como alterações circulatórias sanguíneas e linfáticas, aumentam a predisposição ao linfedema (Kwan et al., 2010; Nascimento Matheus et al., 2011; Baumann et al., 2013; Dayes et al., 2013; McLaughlin et al., 2013).

O linfedema é uma condição crônica, cuja incidência varia de 13 a 65\%, decorrente de alterações circulatórias linfática e sanguínea, com prejuízo da mobilidade do membro (Mayrovitz et al., 2009; Gärtner et al., 2010; Nascimben Matheus, Guirro, 2011; Paskett et al., 2012; Fu et al., 2013, Karadibak et al., 2015, Grada etal., 2017).

O controle do linfedema secundário ao tratamento do câncer de mama é frequentemente aliado a intitulada terapia física complexa (Mondry et al., 2004; Lasinski et al., 2012). Entre outras características, essa terapia é constituída de cuidados da pele, drenagem linfática manual, exercícios físicos ativos, ativos/resistidos e miolinfocinéticos dos membros superiores, e a compressão com enfaixamento compressivo ou manga elástica (Szuba et al., 2000; Tambour et al., 2014).

A técnica do enfaixamento compressivo que utiliza múltiplas camadas de ataduras podem variar em número, com pressão mínima proximal e máxima na região distal do membro superior, considera a Lei de Laplace que aponta que a pressão diminui quando o raio da circunferência aumenta.

O enfaixamento compressivo do membro afetado pelo linfedema visa o controle do edema, além do incremento circulatório sanguíneo e linfático (Moseley et al., 2007; Vignes et al., 2007; Ezzo et al., 2015; Heinig et al., 2015; Vignes et al., 2017), quando aliado a movimentação do membro (Johansson et al., 2005; Johansson, Piller 2007). 
Entretanto, a confiabilidade inter e intra-examinador relacionada a pressão exercida pela técnica de enfaixamento compressivo no tratamento do linfedema de mulheres sobreviventes do câncer de mama, ainda não estão estabelecidas.

A compressão do enfaixamento com pressões menores $(20-30 \mathrm{mmHg})$, é considerada a melhor prática no tratamento do linfedema quando comparadas à pressões maiores (44-58mmHg), sendo observado o mesmo efeito na redução do volume do membro afetado, demonstrando maior conforto e aderência ao tratamento (Damstra et el., 2009; Partsch et al., 2011). No entanto, a aplicação da técnica no tratamento por diferentes indivíduos pode gerar diferentes resultados na pressão exercida pelo enfaixamento.

O desfecho primário na maioria dos estudos de compressão do linfedema é a alteração do volume do membro, entretanto, é necessário investigar a pressão exercida pelas técnicas de compressão empregada e observar seus efeitos (Franzeck et al., 1997; Olszewski, 2003). Dados de ensaios clínicos randomizados que poderiam dar suporte a diferentes questões relacionadas ao tratamento por compressão do linfedema são escassos e difíceis de analisar, pela ampla diversidade de modalidades de tratamento e parâmetros de resultados mensurados (Partsh et al., 2010; Damstra et al, 2011; Lamprou et al., 2011). A ação da compressão de enfaixamento no linfedema não está fundamentada, isto é, estabelecida para o tratamento de doença venosa (Bunke et al., 2009). A compreensão da especificidade dos efeitos na pressão da técnica na circulação sanguínea, e na funcionalidade do membro, procedimentos terapêuticos utilizados amplamente no tratamento do linfedema, pode influenciar em estratégias de intervenção objetivas e eficazes.

Diante do exposto, o objetivo do estudo foi avaliar a confiabilidade, reprodutibilidade, além da e eficiência funcional e circulatória de diferentes formas de ECF. 
Oyjeciura 


\section{OBJETIVO}

\subsection{Objetivo do primeiro estudo}

Avaliar a reprodutibilidade e confiabilidade inter e intraexaminador da técnica de enfaixamento compressivo funcional.

\subsection{Objetivo do segundo estudo}

Avaliar a eficiência de diferentes técnicas de enfaixamento compressivo funcional, na circulação saguínea arterial e venoso braquial, na pressão exercida pelas técnicas, bem como na funcionalidade do membro afetado pelo linfedema.

\section{HIPÓTESE}

Configura-se como hipótese do primeiro estudo que o manejo do linfedema relacionado ao enfaixamento compressivo funcional é aplicador dependente.

A hipótese do segundo estudo, baseia-se na interferência distinta das diferentes formas de aplicação das técnicas de enfaixamento compressivo na circulação sanguínea, na pressão exercidas pelo enfaixamento, bem como na funcionalidade do membro. 
Materiale Malados 


\section{MATERIAL E MÉTODOS}

\subsection{Aspectos éticos}

Os procedimentos do estudo foram aprovados pelo Núcleo de Ensino Pesquisa e Assistência na Reabilitação de Mastectomizadas (REMA) da Escola de Enfermagem de Ribeirão Preto EERP/USP, onde foi desenvolvido o estudo com a aprovação do Comitê de Ética em Pesquisa do Hospital das Clínicas de Ribeirão Preto, processo HCRP $\mathrm{n}^{\circ}$ 6878/2018; CAAE: 90238218.7.0000.5440. O segundo estudo intervencional foi registrado no ClinicalTrials.gov (NCT04076033).

As voluntárias foram informadas de modo prévio sobre a conduta que seriam submetidas, concordando em participar assinaram o Termo de Consentimento Livre e Esclarecido (APÊNDICE 1), efetuado de acordo com a resolução 466/2012 o Conselho Nacional de Saúde (CNS).

Todos os itens foram lidos para as voluntárias durante a avaliação inicial, sendo que os dados obtidas estão arquivadas na Faculdade de Medicina de Ribeirão Preto USP/SP, sob a guarda do coordenador do projeto.

\subsection{Tipo de pesquisa e delineamento do estudo}

O tema foi subdividido em dois diferentes estudos, sendo o primeiro um estudo de reprodutibilidade e confiabilidade do enfaixamento compressivo funcional, e o segundo trata-se de um estudo de intervenção randomizado, cego, tipo crossover, sendo um pesquisador responsável pelo recrutamento e avaliações, outro pela randomização, ainda um terceiro pesquisador responsável pela intervenção dos procedimentos, enquanto um último responsável pelo processamento e análise dos dados coletados. 


\subsection{Amostra}

O processamento do cálculo amostral foi realizado por meio do software Ene, versão 3.0 (Universidade Autônoma de Barcelona, Barcelona, Espanha). A amostra foi calculada com base no estudo Terwee et al., 2007 de confiabilidade, resultando em 50 mulheres para o estudo de confiabilidade e reprodutibilidade, com perda amostral de cinco mulheres, totalizando em 45 mulheres.

Para o estudo de intervenção o cálculo do tamanho da amostra teve como base no estudo de Fong et al. (2014) sendo o principal desfecho a circulação sanguínea, e resultou em 23 mulheres, considerando-se poder estatístico de $90 \%$ e alfa de 0.05 foi estimado um número de 18 mulheres, e considerando-se perdas amostrais, foram avaliadas 23 mulheres.

Participaram do primeiro estudo, 45 mulheres submetidas a cirurgia do câncer de mama que desenvolveram linfedema, com média de idade de 64.88(DP=10.01) anos e índice de massa corporal (IMC) médio de $31.94(\mathrm{DP}=6.05) \mathrm{kg} / \mathrm{m}^{2}$. Foram submetidas a dissecção axilar 28 mulheres, outras 31 mulheres foram submetidas a radioterapia e 33 mulheres a quimioterapia. A diferença média de volume entre os membros foi de 0.35(DP=0.11) $\mathrm{cm}^{3}$, sendo 22 mulheres com linfedema afetando o membro superior direto e 23 o membro superior esquerdo (Tabela 1), e perda amostral de cinco mulheres.

Tabela 1. Características gerais das mulheres do primeiro do estudo de confiabilidade do enfaixamento compressivo funcional $(n=45)$.

\begin{tabular}{lc}
\hline \multicolumn{1}{c}{ Variáveis } & Média \pm Desvio padrão \\
\hline Idade $($ anos) & $64.88 \pm 10.01$ \\
IMC $\left(\mathrm{Kg} / \mathrm{m}^{2}\right)$ & $31.94 \pm 6.05$ \\
Tempo de diagnóstico (meses) & $112.40 \pm 85.75$ \\
Diferença do volume dos membros $\left(\mathrm{cm}^{3}\right)$ & $0,35 \pm 0,11$ \\
\hline Lado da cirurgia & $49 \%$ Destras $-51 \%$ Sinistras \\
Linfadenectomia axilar & $71 \%$ \\
Linfonodo sentinela & $29 \%$ \\
Quimioterapia & $73 \%$ \\
Radioterapia & $68 \%$ \\
\hline
\end{tabular}


Para o segundo estudo, participaram 23 mulheres submetidas a cirurgia do câncer de mama que desenvolveram linfedema, com média de idade de 64.54(DP=11.89) anos e índice de massa corporal (IMC) médio de $32.23(\mathrm{DP}=6.57) \mathrm{kg} / \mathrm{m}^{2}$ (Tabela 2). Todas as mulheres foram submetidas a dissecção axilar, 15 mulheres foram submetidas a radioterapia, e 18 mulheres a quimioterapia. A diferença média de volume entre os membros foi de $0.33(\mathrm{DP}=0.14) \mathrm{cm}^{3}$, sendo 10 mulheres apresentaram linfedema envolvendo o membro superior direito, e 13 o membro superior esquerdo (Tabela 2), sendo que não houve exclusão ou perda amostral.

Tabela 2. Características gerais das mulheres do segundo estudo da eficiência das diferentes técnicas do enfaixamento compressivo funcional $(\mathrm{n}=23)$.

\begin{tabular}{lc}
\hline \multicolumn{1}{c}{ Variáveis } & Média \pm Desvio padrão \\
\hline Idade $(\operatorname{anos})$ & $64.54 \pm 11.89$ \\
IMC $\left(\mathrm{Kg} / \mathrm{m}^{2}\right)$ & $32.23 \pm 6.57$ \\
ITB & $1.03 \pm 0.06$ \\
Tempo de diagnóstico (meses) & $86.43 \pm 71.48$ \\
Diferença do volume dos membros $\left(\mathrm{cm}^{3}\right)$ & $0,33 \pm 0,14$ \\
\hline Membro Dominante & $83 \%$ Destras $-17 \%$ Sinistras \\
Lado da cirurgia & 43\% Destras $-57 \%$ Sinistras \\
Linfadenectomia axilar & $100 \%$ \\
Linfonodo sentinela & $52 \%$ \\
Quimioterapia & $78 \%$ \\
Radioterapia & $65 \%$ \\
\hline
\end{tabular}

\subsection{Critérios de Inclusão}

Foram considerados como critérios de inclusão, idade acima de 21 anos; tratamento para câncer de mama unilateral, considerou-se linfedema unilateral decorrente do tratamento da doença.

\subsection{Critérios de Exclusão}


Os critérios de exclusão estabelecidos foram para que não houvesse influência nos dados de circulação sanguínea e de funcionalidade, ou que agravasse o acometimento em doenças prévias ou ainda que estes alterassem os resultados. Sendo estes considerados, afecções cutâneas, diabetes, tratamento em curso de quimioterapia e radioterapia, esvaziamento axilar bilateral, diagnóstico de metástase e risco de doença arterial periférica, alteração funcional dos membros superiores antes do tratamento do câncer de mama.

\subsection{Avaliação inicial}

A avaliação clínica para os dois estudos incluiu a anamnese padrão constando a partir de dados pessoais das voluntárias, doenças associadas, prática de atividade física, dados relacionados ao tratamento do câncer de mama, uso de medicamentos, além de exame físico com mensuração do índice de massa corporal. Essas variavéis foram estabelecidas para o controle do percurso do tratamento da voluntária.

Para diagnóstico do linfedema, foi avaliada a perimetria do membros superiores, por um avaliador familiarizado com o procedimento (Johnson et al., 2016). As circuferências dos membros foram avaliadas com trena equipada com peso e mola (WCS, Curitiba PR, Brasil), com intuito de padronização da pressão de medida. Designou-se a medida de sete pontos ao longo do membro superior (Sander et al., 2002), Figura 1.

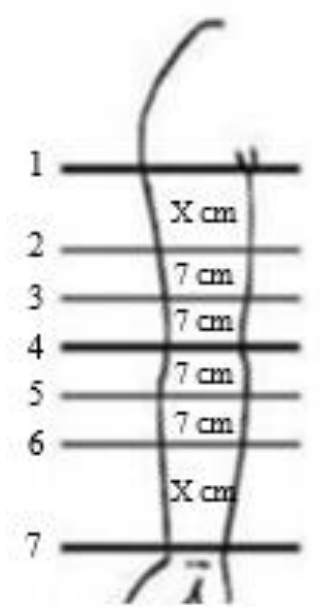

Figura 1. Imagem ilustrativa da mensuração da perimetria. Fonte: Arquivo pessoal. 
Os dados obtidos pela perimetria, possibilitaram o cálculo do volume do membro, considerado "padrão ouro" para aferir edemas irregulares (Petrek et al., 2000), pela fórmula do cone truncado, por método indireto, obtido pela soma do volume dos seis cones truncados, formado entre os sete pontos de medidas das circunferências do braço e antebraço. A soma das seis partes proporciona o volume total do membro (Sander et al., 2002).

$$
V=\frac{1}{12 \pi} \sum_{i=l}^{n} L\left(C_{i}^{2}+C_{i} C_{i-l}+C_{i-l^{2}}\right)
$$

$\mathrm{V}=$ Volume de segmento do membro, $\mathrm{L}=$ comprimento de cada segmento, $C_{i}$ e $C_{i-l}=$ circunferência em cada segmento i.

A confiabilidade intra e inter-examinadores possui bons níveis do método de medida do volume indireto, com valores de coeficiente de correlação intraclasse (ICC) de 0.99 (Sander et al., 2002). O comprometimento do membro afetado por linfedema foi avaliado por meio de critérios estabelecidos por Andersen et al. (2000), e que considera linfedema a diferença entre membros maior ou igual a $200 \mathrm{~mL}$. A classificação do linfedema em leve, moderado e severo foi efetuada por meio de critérios estabelecidos pelo Consenso de 2016 da Sociedade Internacional Linfologia.

\subsection{Primeiro estudo - Confiabilidade do enfaixamento compressivo funcional}

A reprodutibilidade e confiabilidade interexaminador e intraexaminador do enfaixamento compressivo funcional foi avaliada por meio das medidas da pressão exercida por dois examinadores treinados e familiarizados com a técnica, em momentos distintos, com respectivo intervalo de uma semana. (Portney et al., 2000).

As voluntárias foram submetidas ao enfaixamento compressivo funcional pelo primeiro avaliador, sendo avaliada a pressão exercida pelo enfaixamento e, em seguida, um segundo avaliador efetuou o mesmo procedimento. $\mathrm{O}$ registro dos dados foi efetuado 
pelo terceiro examinador em formulários independentes, com intuito de evitar a possível comparação entre os dados durante a coleta dos mesmos.

O enfaixamento compressivo funcional (ECF) em espiral foi efetuado com a paciente sentada, com o membro superior afetado pelo linfedema apoiado em um suporte. Após a hidratação do membro, foi envolvido com uma malha de algodão para evitar o atrito da faixa de espuma de $1 \mathrm{~cm}$ de altura com $28 \mathrm{~g} / \mathrm{cm}$ de densidade. Não foi efetuado nenhum procedimento terapêutico antes do ECF.

O enfaixamento do membro foi efetuado com ataduras elásticas de crepe (Neve ${ }^{\circledR}$ Ind. e Com. Mat. Cir. Ltda., Bragança Paulista/SP, Brasil) com 5 cm, $10 \mathrm{~cm}$, e $15 \mathrm{~cm}$ desde os dedos até a região axilar em espiral com quatro camadas (Randheer et al., 2011). A graduação da pressão exercida pelo enfaixamento foi aferida após o término do enfaixamento por um equipamento desenvolvido para este fim (Rezende et al., 2017), baseado em Damstra et al., 2009 e Kang et al., 2012, posicionados na região de braço e antebraço (Figura 2), e em seguida o ECF era removido.

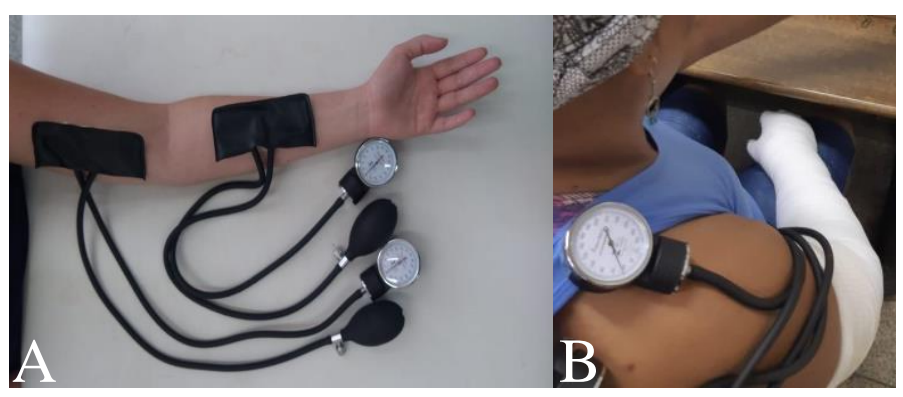

Figura 2. Equipamento para mensuração da pressão, composto por uma bolsa inflável de borracha conectada a um manômetro (A). Posicionamento do equipamento após enfaixamento (B). Fonte: Arquivo pessoal. 


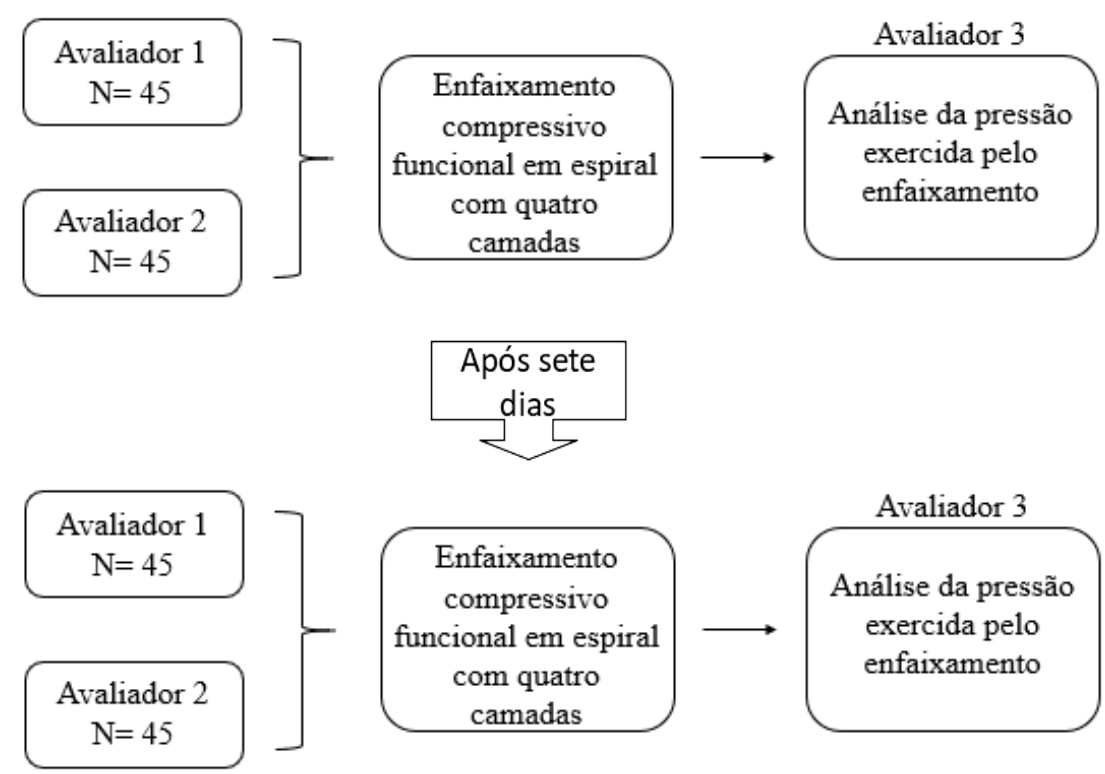

Figura 3. Fluxograma do primeiro estudo da confiabilidade do enfaixamento compressivo funcional.

\subsection{Segundo estudo - Eficiência do enfaixamento compressivo funcional}

Foram avaliadas 23 voluntárias com base no estudo Fong et al., 2014, submetidas a um dos dois procedimentos terapêuticos de enfaixamento compressivo funcional (ECF) padronizados, aplicados por um avaliador, mediante ao sorteio, invertendo a técnica (crossover): técnica em espiral com quatro camadas de ataduras, e técnica em oito com quatro camadas de ataduras, sendo analisada a pressão exercida nas diferentes técnicas de ECF, a funcionalidade do membro superior por meio do teste de Jebsen Taylor com as diferentes técnicas de enfaixamento compressivo, bem como os efeitos na circulação sanguínea, por meio do Doppler, antes e após o teste de Jebsen Taylor. 


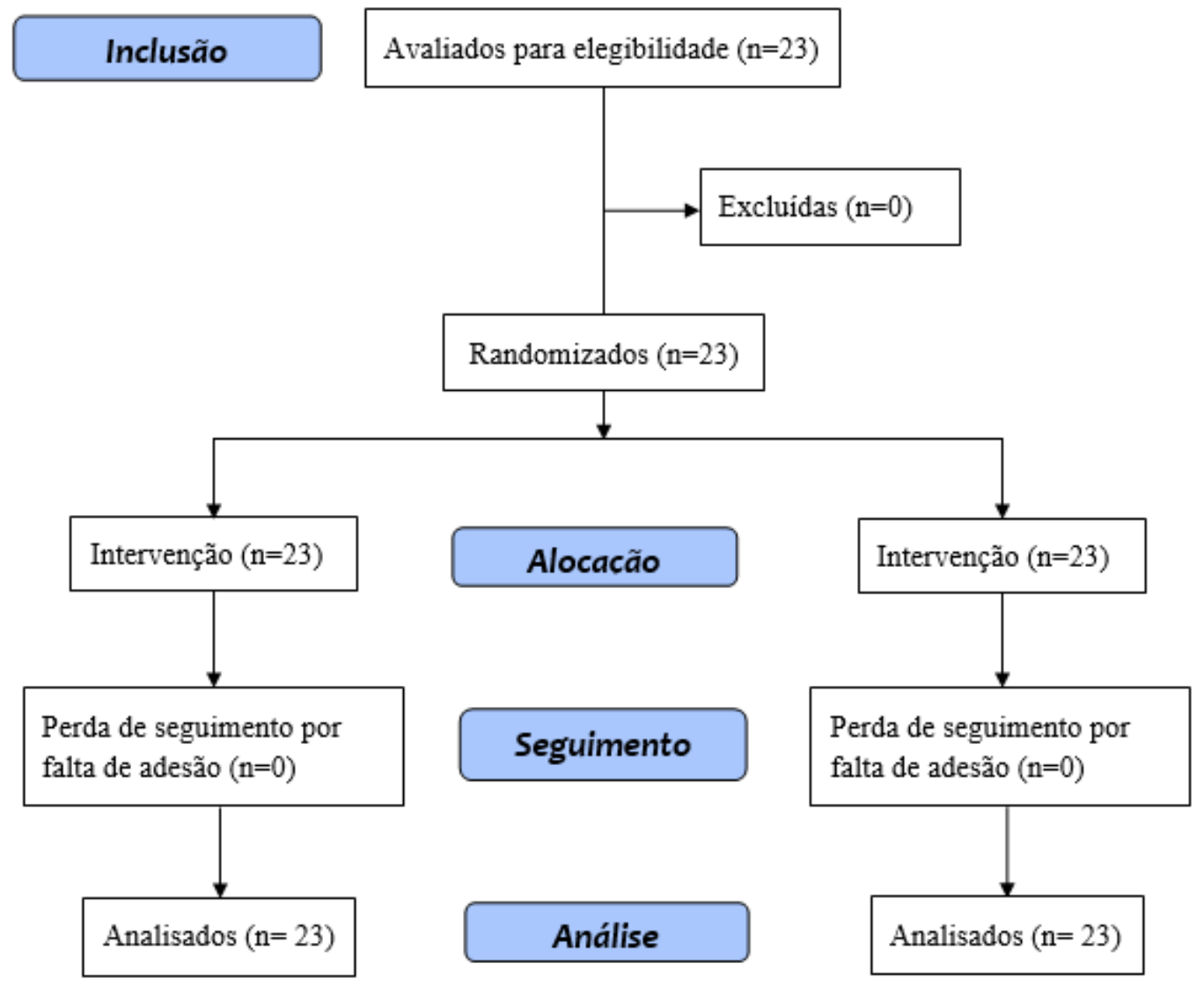

Figura 4. Fluxograma do segundo estudo da eficiência do enfaixamento compressivo funcional

\subsubsection{Enfaixamento compressivo funcional}

O enfaixamento compressivo funcional (ECF) foi efetuado com a paciente sentada com o membro superior afetado pelo linfedema apoiado em um suporte, por apenas um avaliador treinado e familiarizado com a técnica. Após a hidratação do membro, foi envolvido com uma malha de algodão para evitar o atrito da faixa de espuma de $1 \mathrm{~cm}$ de altura com 28 $\mathrm{g} / \mathrm{cm}$ de desndidade. Não foi efetuado nenhum procedimento terapêutico antes do ECF.

O enfaixamento do membro foi efetuado com ataduras elásticas de algodão crepe (Neve ${ }^{\circledR}$ Ind. e Com. Mat. Cir. Ltda., Bragança Paulista/SP, Brasil), com 5 cm, 10 cm, e 15 cm, desde os dedos até a região axilar em quatro camadas (Randheer et al., 2011), aplicados em duas técinas diferentes: "em oito" e "em espiral".

A graduação da pressão exercida pelo enfaixamento foi aferida após o término do enfaixamento e durante o movimento na execução do teste de funcionalidade de Jebsen 
Taylor, por um equipamento desenvolvido para este fim (Rezende et al., 2017), baseado em Damstra et al., 2009 e Kang et al., 2012, posicionados na região de braço e antebraço (Figura 2). Durante o movimento, a pressão exercida pelo ECF somava a contração muscular, apresentando variação de pressão miníma e máxima.

\subsubsection{Avaliação do fluxo sanguíneo}

A avaliação da circulação arterial e venosa braquial foi efetuada por meio de ultrassom Doppler SONARA/Tek (Nicolet Vascular-Madison, USA), com probe de 4 MHz. A análise do fluxo sanguíneo, por sua vez, foi efetuada com o transdutor posicionado a $45^{\circ} \mathrm{C}$ em relação ao vaso sanguíneo avaliado, com a pele untada com gel solúvel em água. A confirmação da localização apropriada para avaliação detectada por meio de observação da imagem espectral e sinais sonoros produzidos pelo equipamento.

Para avaliação do fluxo sanguíneo, as voluntárias premaneceram em repouso durante 5 minutos em decúbito dorsal, o membro foi avaliado em posição supina na fossa cubital, em sala com temperatura controlada em $23^{\circ} \mathrm{C}$ (Beckman et al., 2001).

Notou-se que o comprometimento arterial geral foi avaliado pelo índice tornozelo braço (ITB), com ultrassom Doppler SONARA/Tek (Nicolet Vascular-Madison, USA), e esfignomanômetro Premium (Accumed, Duque de Caxias - RJ), as medidas avaliadas foram em decúbito dorsal, após 5 minutos de repouso, sendo a medida pressão sistólica da artéria tibial posterior e da artéria braquial bilateralmente.

O ITB foi obtido por meio da razão entre a maior das pressões no tornozelo pela maior pressão sistólica identificada na artéria braquial dos membros superiores (Nogren et al., 2007). Os valores considerados normais para o ITB estão entre 0,9 e 1,4, sendo os índices maiores que 1,4 demonstram aumento da resistência das artérias e índices menores ou iguais a 0,9 representam a presença de doença arterial periférica, DAP (Nogren et al., 2007). 


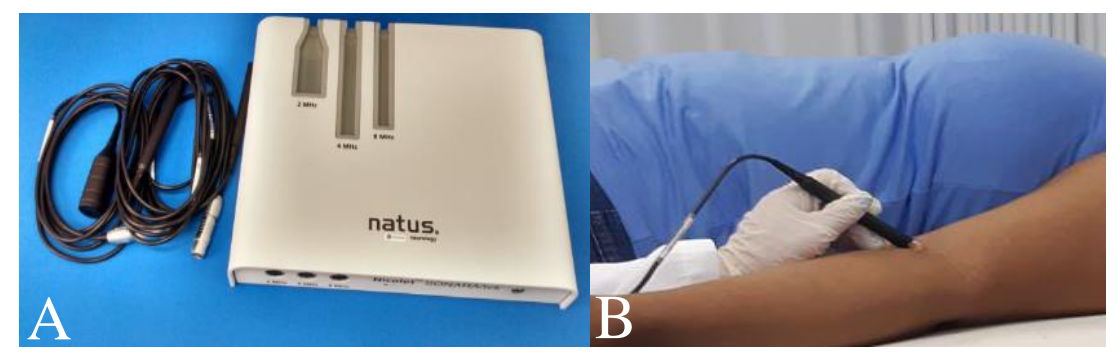

Figura 5 - Equipamento de ultrassom Doppler SONARA/Tek (A). Avaliação da circulação sanguínea (B). Fonte: Arquivo pessoal.

\subsubsection{Teste de função manual Jebsen Taylor}

Com o desenvolvimento prático em foco, foi possível perceber que a dominância do membro superior foi verificada por meio do inventário de Edinburgh (Brito et al., 1989), antes da aplicação do teste de funcionalidade Jebsen Taylor (TFMJT).

O TFMJT é constituído por sete subtestes que mimetizam atividades de vida diária como a escrita, manipular cartas, levantar objetos pequenos, simular a alimentação, empilhar blocos, levantar objetos grandes e leves e levantar de objetos grandes e pesados.

O tempo foi registrado por um cronômetro digital (TS-1809-Taksun, China) para cada tarefa executada, e o desempenho avaliado por meio da somatória dos tempos gastos em cada um deles (Lima et al., 2012).

O teste de Jebsen Taylor é considerado como uma medida confiavél para classificar o desempenho de disfunções dos membros superiores na populações da Itália, e para pessoas com distrofia muscular (Culicchia et al., 2016; Artilheiro et al., 2018). No entanto, ainda não há uma validação desse método em mulheres com câncer de mama. 


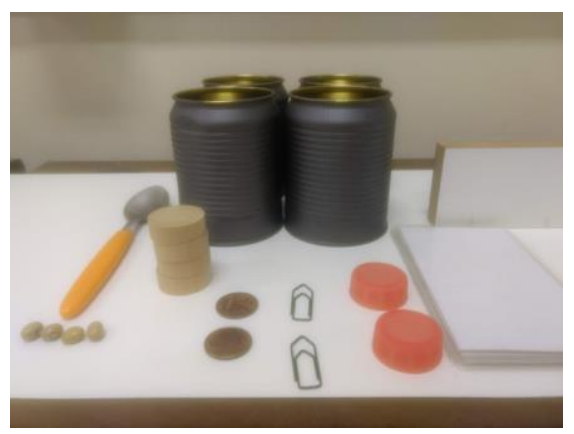

Figura 6 - Itens do teste de funcionalidade Jebsen Taylor, composto por diferentes objetos. Fonte: Arquivo pessoal.

\subsection{Análise estatística}

A análise da confiabilidade inter e intraexaminadores e da reprodutibilidade do método foi testada pelo coeficiente de correlação intraclasse (CCI), com intervalo de confiança de 95\%, diferença mínima detectável (DMD) e erro padrão da medida (EPM) de acordo com critérios estabelecidos por Weir (2005). A interpretação do valor do CCI foi baseada no estudo de Fleiss (1986): para valores abaixo de 0,40, a confiabilidade foi considerada baixa; entre 0,40 e 0,75 moderada; entre 0,75 e 0,90, alto, e para valores maiores que 0,90 , a confiabilidade foi considerada excelente.

O teste de normalidade de Shapiro-Wilk foi aplicado paraobservação da distribuição dos dados, e testes estatísticos condizentes (Anova com post-hoc de Tukey ou Kruskal-Walis com post-hoc de Dunn) para comparações intra e inter entre os grupos, considerando-se dois fatores nas comparações, tempo e grupo.

Considerou-se um modelo de regressão linear com efeitos mistos para a análise das variáveis, e aleatório por indivíduo em diversas medidas diferentes, considerando o efeito fico a independência entre cada indivíduo. O processamento dos dados foi efetuado por SAS Statistical Software (version 9.3; SAS Institute, Inc. Cary, NC), considerando um intervalo de confiança de $95 \%$. 
Gresultados 


\section{RESULTADOS}

\subsection{Resultados referentes à confiabilidade do enfaixamento compressivo funcional}

A Tabela 3 exibe resultados da reprodutibilidade e confiabilidade interexaminador na região de braço e antebraço, no primeiro avaliador revelou que a região de braço a confiabilidade foi considerada baixa (CCI: 0.2 ), e na região de antebraço foi considerada moderada (CCI: 0.6). Para o segundo avaliador, ambas as regiões de braço e antebraço foram consideradas com uma confiabilidade baixa (CCI: -0.3; -0.2).

Os resultados da Tabela 4 demonstram que a reprodutibilidade e confiabilidade intraexaminador na região de braço e antebraço para o avaliador A, em que foi considerada de confiabilidade baixa (CCI: -0.04; -0.3). Já para o avaliador B, a confibilidade na região de braço foi considerada alta (CCI: 0.8), e na região de antebraço foi considerada confiabilidade moderada (CCI: 0.7$)$.

Tabela 3. Resultados da reprodutibilidade e confiabilidade interexaminador da técnica de enfaixamento compressivo funcional, modalidade em espiral. $n=45$.

\begin{tabular}{|l|c|c|}
\hline \multirow{2}{*}{ Variavéis } & $1^{\circ}$ Avaliador & $2^{\circ}$ Avaliador \\
\cline { 2 - 3 } & CCI (95\% IC) & CCI (95\% IC) \\
\hline Braço & $0.2(-0.4 ; 0.6)$ & $-0.3(-1.4 ; 0.2)$ \\
Antebraço & $0.6(0.2 ; 0.8)$ & $-0.2(-1.1 ; 0.4)$ \\
\hline
\end{tabular}

CCI: coeficiente de correlação intraclasse; 95\% IC: intervalo de confiança de $95 \%$.

Tabela 4. Resultados da reprodutibilidade e confiabilidade intraexaminador da técnica de enfaixamento compressivo funcional, modalidade em espiral. $n=45$.

\begin{tabular}{|l|c|c|}
\hline \multirow{2}{*}{ Variavéis } & Avaliador A & Avaliador B \\
\cline { 2 - 3 } & CCI (95\% IC) & CCI (95\% IC) \\
\hline Braço & $-0.04(-0.9 ; 0.4)$ & $0.8(0.7 ; 0.9)$ \\
Antebraço & $-0.3(-1.5 ; 0.3)$ & $0.7(0.4 ; 0.8)$ \\
\hline
\end{tabular}

CCI: coeficiente de correlação intraclasse; 95\% IC: intervalo de confiança de $95 \%$. 


\subsection{Comparações do fluxo e pico sanguíneo sem e com enfaixamento compressivo funcional}

A tabela 5 aponta os resultados de média (desvio padrão) e o comportamento do fluxo e pico sanguíneo arterial e venoso no membro afetado pelo linfedema, em três situações (repouso, após o teste de Jebsen Taylor sem e com enfaixamento compressivo funcional), e com duas técnicas de enfaixamento compressivo funcional (ECF) distintas (espiral e oito).

A compração seguiu a ordem: repouso e após o teste de Jebsen Taylor, sem o enfaixamento compressivo funcional (SECF), houve repouso e em seguida, o teste de Jebsen Taylor com o ECF (CECF); logo após o teste de Jebsen Taylor sem o ECF (SECF) e posteriormente o teste de Jebsen Taylor com ECF (CECF), nessa respectiva ordem.

Houve um aumento significativo na velocidade média e pico do fluxo arterial e venoso observado após o teste de Jebsen Taylor sem ECF ( $\mathrm{p}<0.05)$ quando comparado ao repouso, o mesmo foi observado após o teste de Jebsen Taylor com ECF $(\mathrm{p}<0.05)$ quando comparado ao repouso e após o teste de Jebsen Taylor sem ECF, isso ocorreu nas duas técnicas de ECF (espiral e oito).

A Tabela 6 exibe os resultados referentes ao teste de funcionalidade de Jebsen Taylor com diferentes técnicas ECF (espiral e oito), sendo que a técnica em oito apresentou um aumento significativo na velocidade do fluxo arterial $(\mathrm{p}=0.0071)$, quando comparado com a técnica em espiral. Não houve diferenças significativas no pico do fluxo arterial, e na velocidade média e pico do fluxo venoso. 
Tabela 5. Média (desvio padrão) das variáveis avaliadas e a comparação entre as situações no ultrassom Doppler $(\mathrm{cm} / \mathrm{s})$ analisadas, velocidade média do fluxo arterial e venoso, e pico do fluxo arterial e venoso do vaso braquial, no membro superior afetado, em repouso e após o teste de função manual sem e com enfaixamento compressivo funcional( espiral e em oito). $n=23$.

\begin{tabular}{|c|c|c|c|c|c|c|c|}
\hline \multirow{2}{*}{ Variáveis } & \multirow{2}{*}{$\begin{array}{c}\text { Tipo } \\
\text { Enfaixamento }\end{array}$} & \multirow[t]{2}{*}{ Situação } & \multirow{2}{*}{$\begin{array}{l}\text { Média (desvio } \\
\text { padrão) }\end{array}$} & \multicolumn{2}{|c|}{ Comparação } & \multirow{2}{*}{$\begin{array}{l}\text { Diferença } \\
\text { Estimada }\end{array}$} & \multirow{2}{*}{ Valor $\mathrm{p}$} \\
\hline & & & & Situação & Situação & & \\
\hline $\begin{array}{l}\text { Velocidade média } \\
\text { arterial }\end{array}$ & Espiral & Repouso & $19.08( \pm 7.40)$ & Repouso & SECF & $-2,9987$ & $0,0164 *$ \\
\hline $\begin{array}{c}\text { Velocidade média } \\
\text { arterial }\end{array}$ & Espiral & SECF & $22.08( \pm 7.94)$ & Repouso & $\mathrm{CECF}$ & $-7,0248$ & $0,0001 *$ \\
\hline $\begin{array}{c}\text { Velocidade média } \\
\text { arterial }\end{array}$ & Espiral & CECF & $26.1( \pm 8.59)$ & SECF & $\mathrm{CECF}$ & $-4,0261$ & $0,0013^{*}$ \\
\hline $\begin{array}{c}\text { Velocidade média } \\
\text { arterial }\end{array}$ & Oito & Repouso & $17.72( \pm 4.86)$ & Repouso & SECF & $-5,2322$ & $0,0001^{*}$ \\
\hline $\begin{array}{c}\text { Velocidade média } \\
\text { arterial }\end{array}$ & Oito & SECF & $22.95( \pm 6.25)$ & Repouso & CECF & $-11,7583$ & $0,0001^{*}$ \\
\hline $\begin{array}{c}\text { Velocidade média } \\
\text { arterial }\end{array}$ & Oito & $\mathrm{CECF}$ & $29.47( \pm 9.09)$ & SECF & CECF & $-6,5261$ & $0,0001 *$ \\
\hline Pico do fluxo arterial & Espiral & Repouso & $74,99( \pm 20.68)$ & Repouso & SECF & & $0,0485^{*}$ \\
\hline Pico do fluxo arterial & Espiral & SECF & $82,73( \pm 18.06)$ & Repouso & CECF & $-17,5087$ & $0,0001 *$ \\
\hline Pico do fluxo arterial & Espiral & CECF & $92,5( \pm 18.57)$ & SECF & CECF & $-9,7739$ & $0,0129 *$ \\
\hline Pico do fluxo arterial & Oito & Repouso & $75,96( \pm 18.40)$ & Repouso & SECF & $-15,0478$ & $0,0001 *$ \\
\hline Pico do fluxo arterial & Oito & SECF & $91,01( \pm 19.34)$ & Repouso & CECF & $-23,6739$ & $0,0001 *$ \\
\hline Pico do fluxo arterial & Oito & $\mathrm{CECF}$ & $99,63( \pm 16.34)$ & SECF & $\mathrm{CECF}$ & & $0,0279 *$ \\
\hline $\begin{array}{l}\text { Velocidade média } \\
\text { venoso }\end{array}$ & Espiral & Repouso & $11,65( \pm 4.11)$ & Repouso & SECF & $-3,5652$ & $0,0036^{*}$ \\
\hline $\begin{array}{l}\text { Velocidade média } \\
\text { venoso }\end{array}$ & Espiral & SECF & $11,36( \pm 4.25)$ & Repouso & $\mathrm{CECF}$ & $-9,7826$ & $0,0001 *$ \\
\hline $\begin{array}{l}\text { Velocidade média } \\
\text { venoso }\end{array}$ & Espiral & $\mathrm{CECF}$ & $11,88( \pm 4.66)$ & SECF & CECF & $-6,2174$ & $0,0001^{*}$ \\
\hline $\begin{array}{l}\text { Velocidade média } \\
\text { venoso }\end{array}$ & Oito & Repouso & $10,78( \pm 4.53)$ & Repouso & SECF & $-4,1304$ & $0,0012^{*}$ \\
\hline $\begin{array}{l}\text { Velocidade média } \\
\text { venoso }\end{array}$ & Oito & SECF & $11,53( \pm 4.90)$ & Repouso & CECF & $-10,6957$ & $0,0001^{*}$ \\
\hline $\begin{array}{c}\text { Velocidade média } \\
\text { venoso }\end{array}$ & Oito & CECF & $12,35( \pm 5.31)$ & SECF & $\mathrm{CECF}$ & $-6,5652$ & $0,0001^{*}$ \\
\hline Pico do fluxo venoso & Espiral & Repouso & $13,85( \pm 3.87)$ & Repouso & SECF & $-4,0000$ & $0,0038^{*}$ \\
\hline Pico do fluxo venoso & Espiral & SECF & $14,2( \pm 5.16)$ & Repouso & $\mathrm{CECF}$ & $-9,4783$ & $0,0001 *$ \\
\hline Pico do fluxo venoso & Espiral & CECF & $14,22( \pm 4.75)$ & SECF & $\mathrm{CECF}$ & $-5,4783$ & $0,0001 *$ \\
\hline Pico do fluxo venoso & Oito & Repouso & $13,16( \pm 5.43)$ & Repouso & SECF & $-4,1304$ & $0,0024 *$ \\
\hline Pico do fluxo venoso & Oito & SECF & $13,88( \pm 5.43)$ & Repouso & CECF & $-9,7826$ & $0,0001^{*}$ \\
\hline Pico do fluxo venoso & Oito & $\mathrm{CECF}$ & $14,61( \pm 4.91)$ & SECF & $\mathrm{CECF}$ & $-5,6522$ & $0,0001 *$ \\
\hline
\end{tabular}

SECF: sem enfaixamento compressivo funcional; CECF: com enfaixamento compressivo funcional. *Diferença significativa $(\mathrm{p}<0.05)$. 
Tabela 6. Resultados da comparação das variáveis do ultrassom Doppler $(\mathrm{cm} / \mathrm{s})$ analisadas, velocidade média do fluxo arterial e venoso, e pico do fluxo arterial e venoso do vaso braquial, no membro superior afetado, após o teste de função manual com enfaixamento compressivo funcional (espiral e em oito). $\mathrm{n}=23$.

\begin{tabular}{|ccccc|}
\hline \multirow{2}{*}{ Variáveis } & \multicolumn{2}{c}{ Comparação } & Diferença & Valor $\mathrm{p}$ \\
\cline { 2 - 4 } & $\begin{array}{c}\text { Tipo } \\
\text { Enfaixamento }\end{array}$ & $\begin{array}{c}\text { Tipo } \\
\text { Enfaixamento }\end{array}$ & Estimada & \\
\hline Velocidade média arterial & Espiral & Oito & $-3,3696$ & $0,0071^{*}$ \\
\hline Pico do fluxo arterial & Espiral & Oito & $-7,1348$ & 0,0686 \\
\hline Velocidade média venoso & Espiral & Oito & $-0,4783$ & 0,3515 \\
\hline Pico do fluxo venoso & Espiral & Oito & 0,3478 & 0,6544 \\
\hline
\end{tabular}

* Diferença significativa $(\mathrm{p}<0.05)$. 
5.3. Comparação da pressão exercida pelo enfaixamento compressivo funcional com diferentes técnicas (espiral e oito)

A Tabela 7 aponta os resultados de média (desvio padrão), e a comparação entre as técnicas de enfaixamento compressivo funcional (ECF) em espiral e oito, na pressão exercida pelo ECF e sua variação (mínimo e máximo) na região de braço e antebraço, durante o repouso e movimento.

Não foi observada diferença significativa na pressão exercida entre a técnica em oito e espiral no repouso. Foi observada uma pressão significativamente maior na técnica em oito quando comparado a técnica em espiral durante o movimento nas regiões de braço e antebraço $(\mathrm{p}<0.05)$. 
Tabela 7. Média (desvio padrão) das variáveis avaliadas e a comparação da pressão (mmHg) exercida pelo enfaixamento compressivo funcional em espiral ou em oito sobre o tecido, durante o repouso e o momento, na região de braço e antebraço. $n=23$.

\begin{tabular}{|c|c|c|c|c|c|c|c|c|c|}
\hline \multirow[b]{2}{*}{ Local } & \multirow[b]{2}{*}{ Situação } & \multicolumn{4}{|c|}{ Enfaixamento } & \multicolumn{4}{|c|}{ Comparação } \\
\hline & & $\begin{array}{c}\text { Espiral } \\
\text { Média (DP) }\end{array}$ & $\begin{array}{c}\text { Espiral } \\
\text { (Mínimo- } \\
\text { Máximo) }\end{array}$ & $\begin{array}{c}\text { Oito } \\
\text { Média (DP) }\end{array}$ & $\begin{array}{c}\text { Oito } \\
\text { (Mínimo- } \\
\text { Máximo) }\end{array}$ & $\mathrm{ECF}$ & $\mathrm{ECF}$ & $\begin{array}{l}\text { Diferença } \\
\text { Estimada }\end{array}$ & Valor $\mathrm{p}$ \\
\hline Braço & Repouso & $33.7( \pm 8.42)$ & $20-50$ & $38.04( \pm 6.17)$ & $30-50$ & espiral & oito & $-4,35$ & 0,0743 \\
\hline Braço & Mínimo & $53.91( \pm 8.39)$ & $40-70$ & $59.13( \pm 7.33)$ & $40-70$ & espiral & oito & $-5,22$ & $0,0324 *$ \\
\hline Braço & Máximo & $89.57( \pm 15.81)$ & $60-120$ & $104.3( \pm 14.17)$ & $100-120$ & espiral & oito & $-14,78$ & $0,0001^{*}$ \\
\hline Antebraço & Repouso & $38.26( \pm 7.01)$ & $20-50$ & $42.61( \pm 8.51)$ & $30-60$ & espiral & oito & $-4,35$ & 0,0743 \\
\hline Antebraço & Mínimo & $56.09( \pm 7.22)$ & $40-70$ & $61.3( \pm 9.68)$ & $40-80$ & espiral & oito & $-5,22$ & $0,0324 *$ \\
\hline Antebraço & Máximo & $84.35( \pm 11.99)$ & $60-110$ & $98.04( \pm 12.13)$ & $70-130$ & espiral & oito & $-13,70$ & $0,0001^{*}$ \\
\hline
\end{tabular}

DP: desvio padrão; Mínimo: mínimo de pressão exercida pelo enfaixamento compressivo funcional durante o movimento; Máximo: máximo de pressão exercida pelo enfaixamento compressivo funcional durante o movimento; ECF: enfaixamento compressivo funcional. 
5.4. Comparação do teste de funcionalidade de Jebsen Taylor com e sem enfaixamento compressivo funcional

A Tabela 8 apresenta os valores de média (desvio padrão) e a comparação de sete variáveis do teste de funcionalidade de Jebsen Taylor, com e sem enfaixamento compressivo funcional (ECF) no membro afetado pelo linfedema.

Foi observado um tempo de execução maior durante o teste com ECF comparado ao teste sem ECF $(\mathrm{p}<0.05)$ nas diferentes técnicas (espiral e oito).

A tabela 9 exibe os resultados da comparação entre as técnicas de ECF, e aponta que não foram encontradas diferenças significativas entre as mesmas. 
Tabela 8. Média (desvio padrão) e a comparação entre as variáveis de Jebsen Taylor sem ou com enfaixamento compressivo funcional em espiral ou em oito no membro superior. $\mathrm{n}=23$.

\begin{tabular}{|c|c|c|c|c|c|c|c|c|}
\hline \multirow{2}{*}{ Variáveis } & \multirow{2}{*}{$\mathrm{ECF}$} & \multirow[b]{2}{*}{ Lado } & \multicolumn{2}{|c|}{ Média (DP) } & \multicolumn{2}{|c|}{ Comparação } & \multirow{2}{*}{$\begin{array}{r}\text { Diferença } \\
\text { Estimada }\end{array}$} & \multirow{2}{*}{ Valor $\mathrm{p}$} \\
\hline & & & SECF & CECF & Situação & Situação & & \\
\hline Escrita & Espiral & MA & $36.68( \pm 26.42)$ & $39.42( \pm 30.11)$ & SECF & $\mathrm{CECF}$ & $-2,7448$ & 0,5953 \\
\hline Escrita & - & MNA & $31.6( \pm 18.37)$ & $30.21( \pm 3.94)$ & SECF & CECF & 1,3839 & 0,7888 \\
\hline Escrita & Oito & MA & $39.76( \pm 31.51)$ & $38.81( \pm 28.04)$ & SECF & CECF & 0,9513 & 0,8539 \\
\hline Escrita & - & MNA & $31.29( \pm 21.23)$ & $30.06( \pm 14.32)$ & SECF & CECF & 1,2261 & 0,8124 \\
\hline Virar cartas & Espiral & MA & $10.52( \pm 4.90)$ & $14.06( \pm 7.52)$ & SECF & CECF & $-3,5361$ & $0,022 *$ \\
\hline Virar cartas & - & MNA & $10.32( \pm 5.59)$ & $9.32( \pm 4.62)$ & SECF & CECF & 0,997 & 0,515 \\
\hline Virar cartas & Oito & MA & $10.37( \pm 4.57)$ & $15.26( \pm 13.30)$ & SECF & CECF & $-4,8883$ & $0,0017 *$ \\
\hline Virar cartas & - & MNA & $10.39( \pm 5.89)$ & $8.74( \pm 4.46)$ & SECF & CECF & 1,6457 & 0,2831 \\
\hline Objetos comuns & Espiral & MA & $10.55( \pm 4.19)$ & $12.7( \pm 4.90)$ & SECF & CECF & $-2,1461$ & $0,0044^{*}$ \\
\hline Objetos comuns & - & MNA & $9.84( \pm 2.77)$ & $10.71( \pm 3.66)$ & SECF & CECF & $-0,8604$ & 0,2478 \\
\hline Objetos comuns & Oito & MA & $10.77( \pm 3.14)$ & $12.97( \pm 5.34)$ & SECF & CECF & $-2,2013$ & $0,0035^{*}$ \\
\hline Objetos comuns & - & MNA & $9.72( \pm 3.41)$ & $9.28( \pm 2.72)$ & SECF & CECF & 0,4387 & 0,5551 \\
\hline $\begin{array}{c}\text { Simular } \\
\text { alimentação }\end{array}$ & Espiral & MA & $9.97( \pm 2.59)$ & $12.64( \pm 3.87)$ & SECF & CECF & $-2,667$ & $0,0005^{*}$ \\
\hline $\begin{array}{c}\text { Simular } \\
\text { alimentação }\end{array}$ & - & MNA & $9.20( \pm 2.74)$ & $8.61( \pm 2.45)$ & SECF & CECF & 0,5917 & 0,4336 \\
\hline $\begin{array}{c}\text { Simular } \\
\text { alimentação }\end{array}$ & Oito & MA & $9.57( \pm 2.87)$ & $12.41( \pm 4.79)$ & SECF & CECF & $-2,8374$ & $0,0002^{*}$ \\
\hline $\begin{array}{c}\text { Simular } \\
\text { alimentação }\end{array}$ & - & MNA & $8.97( \pm 2.57)$ & $9.56( \pm 2.89)$ & SECF & CECF & $-0,59$ & 0,435 \\
\hline $\begin{array}{c}\text { Empilhar } \\
\text { damas }\end{array}$ & Espiral & MA & $5.65( \pm 1.57)$ & $6.82( \pm 2.71)$ & SECF & CECF & $-1,1752$ & $0,002 *$ \\
\hline $\begin{array}{l}\text { Empilhar } \\
\text { damas }\end{array}$ & - & MNA & $5.33( \pm 1.21)$ & $5.25( \pm 1.72)$ & SECF & CECF & 0,07609 & 0,8393 \\
\hline $\begin{array}{l}\text { Empilhar } \\
\text { damas }\end{array}$ & Oito & MA & $6.07( \pm 2.39)$ & $6.53( \pm 2.07)$ & SECF & CECF & $-0,4609$ & 0,2204 \\
\hline $\begin{array}{c}\text { Empilhar } \\
\text { damas }\end{array}$ & - & MNA & $5.3( \pm 1.48)$ & $5.30( \pm 1.68)$ & SECF & CECF & $-0,00174$ & 0,9963 \\
\hline $\begin{array}{c}\text { Levantar } \\
\text { objetos leves }\end{array}$ & Espiral & MA & $3.69( \pm 0.76)$ & $4.28( \pm 1.27)$ & SECF & CECF & $-0,5904$ & $0,0023^{*}$ \\
\hline $\begin{array}{c}\text { Levantar } \\
\text { objetos leves }\end{array}$ & - & MNA & $3.54( \pm 0.82)$ & $3.55( \pm 1.07)$ & SECF & CECF & $-0,00826$ & 0,9655 \\
\hline $\begin{array}{c}\text { Levantar } \\
\text { objetos leves }\end{array}$ & Oito & MA & $3.98( \pm 1.03)$ & $4.49( \pm 1.36)$ & SECF & CECF & $-0,5057$ & $0,0089^{*}$ \\
\hline $\begin{array}{c}\text { Levantar } \\
\text { objetos leves }\end{array}$ & - & MNA & $3.51( \pm 0.74)$ & $3.44( \pm 0.85)$ & SECF & CECF & 0,06826 & 0,721 \\
\hline $\begin{array}{c}\text { Levantar } \\
\text { objetos pesados }\end{array}$ & Espiral & MA & $4.01( \pm 1.15)$ & $4.10( \pm 1.14)$ & SECF & CECF & $-0,09565$ & 0,5483 \\
\hline $\begin{array}{c}\text { Levantar } \\
\text { objetos pesados }\end{array}$ & - & MNA & $3.87( \pm 1.12)$ & $3.60( \pm 0.84)$ & SECF & CECF & 0,2674 & 0,0946 \\
\hline $\begin{array}{c}\text { Levantar } \\
\text { objetos pesados }\end{array}$ & Oito & MA & $4.04( \pm 0.79)$ & $4.37( \pm 0.98)$ & SECF & CECF & $-0,3222$ & $0,0445^{*}$ \\
\hline $\begin{array}{c}\text { Levantar } \\
\text { objetos pesados }\end{array}$ & - & MNA & $3.70( \pm 0.92)$ & $3.72( \pm 0.86)$ & SECF & CECF & $-0,01478$ & 0,926 \\
\hline
\end{tabular}

ECF: enfaixamento compressivo funcional; DP: desvio padrão; MA: membro afetado; MNA: membro não afetado; SECF: sem enfaixamento compressivo funcional; CECF: com enfaixamento compressivo funcional; (-): membro não afetado não efetuou enfaixamento compressivo funcional. 
Tabela 9. Resultados da comparação entre os tipos de enfaixamento compressivo funcional e as variáveis de Jebsen Taylor com enfaixamento compressivo funcional. $n=23$.

\begin{tabular}{|c|c|c|c|c|c|}
\hline \multirow[b]{2}{*}{ Variáveis } & \multirow[b]{2}{*}{ Situação } & \multicolumn{2}{|c|}{ Comparação } & \multirow{2}{*}{$\begin{array}{l}\text { Diferença } \\
\text { Estimada }\end{array}$} & \multirow{2}{*}{$\begin{array}{c}\text { Valor } \\
\mathrm{p}\end{array}$} \\
\hline & & $\begin{array}{c}\text { Tipo } \\
\text { Enfaixamento }\end{array}$ & $\begin{array}{c}\text { Tipo } \\
\text { Enfaixamento }\end{array}$ & & \\
\hline Escrita & CECF & Espiral & Oito & 0,6109 & 0,9059 \\
\hline Virar cartas & CECF & Espiral & Oito & $-1,1965$ & 0,4347 \\
\hline Objetos comuns & $\mathrm{CECF}$ & Espiral & Oito & $-0,267$ & 0,7194 \\
\hline Simular alimentação & CECF & Espiral & Oito & 0,2248 & 0,7659 \\
\hline Empilhar damas & CECF & Espiral & Oito & 0,2891 & 0,4413 \\
\hline Levantar objetos leves & CECF & Espiral & Oito & $-0,2043$ & 0,2858 \\
\hline $\begin{array}{c}\text { Levantar objetos } \\
\text { pesados }\end{array}$ & CECF & Espiral & Oito & $-0,2643$ & 0,0984 \\
\hline
\end{tabular}

CECF: com enfaixamento compressivo funcional. 
- Siéculesaña 


\section{DISCUSSÃO}

O objetivo do primeiro estudo foi avaliar a reprodutibilidade e confiabilidade do enfaixamento compressivo funcional (ECF), que demonstrou ser um método de baixa a moderada confiabilidade interxaminador, e confiabilidade baixa a alta intraexaminadores.

Os achados demonstram a dificuldade de padronização do grau de pressão empregado pela técnica, mesmo que está tenha o número de camadas e a técnica padronizados. O resultado encontrado pode ser explicado pela lei de Laplace, em que a pressão de compressão pode variar de acordo com tensão empregada pela atadura, pelo número de camadas de ataduras e pela heterogeneidade da circunferência do membro (Pellicer et al., 2000). A variação inerente na aplicação da técnica por diferentes aplicadores pode estar relacionada ao estiramento da faixa, fator diretamente relacionado a pressão imposta.

Estudos efetuados com terapias compressivas no membro inferior (Partsh et al., 2008; Partsh et al., 2012; Fu et al., 2014), apontam a dificuldade de padronizar o emprego das técnicas do ECF, além da manutenção da pressão exercida pelo enfaixamento aliado aos movimentos efetuados pelo paciente, sendo que a compressão elástica exerce pressão constante, pelo fato da mesma ser tecnologicamente estabelecida pelos tecidos empregados, além de não ocorrer afrouxamento por escorregamento. Portanto, o presente estudo confirma a dificuldade na padronização da pressão exercida pelo ECF no membro afetado, que depende de diferentes fatores.

Os resultados do presente estudo apontam para a dificuldade de padronização da aplicação da técnica de ECF intra e interexaminador. Estudo realizado (Karakashian et al., 2019) onde investigou a terapia de compressão elástica em 23 mulheres com linfedema no membro superior, com objetivo de interpretar as tensões, deformações de estruturas subcutâneas e cutâneas, e o efeito da forma do membro sobre pressões em cada paciente, encontrou uma variabilidade na pressão entre os membros, a implicação da 
pressão exercida sobre o tecido depende do grau e forma do linfedema.

A compressão no tratamento de edemas é considerada como um método confiável, pois possui nível de evidência Grau B para o tratamento do linfedema, para o Best Practice for the Management of Lymphoedema, (2006), atua com resultados favoráveis na proteção da pele, disfunção circulatória, melhora da contratilidade músculo-vascular, aumento da pressão intersticial, deslocamento de fluidos e na redução no volume do membro (King et al., 2012). Sabe-se os efeitos benéficos da terapia de compressão, entretanto, são necessários mais estudos para auxiliar no planejamento adequado no tratamento compressivo.

O objetivo do segundo estudo foi avaliar os efeitos inerentes a diferentes tipos de ECF na circulação sanguínea do membro afetado por linfedema, bem como a funcionalidade do membro. O comportamento da circulação sanguínea com e sem ECF após teste de função manual de Jebsen Taylor, apontou um incremento circulatório arterial e venoso braquial com ECF, havendo aumento do fluxo sanguíneo após o teste com ECF comparado ao repouso e após o teste sem o ECF. Além disso, o incremento circulatório foi observado em ambos os tipos de técnicas de ECF (espiral e oito), não sendo observada diferenças entre elas.

A pressão de repouso, que é constante sobre a pele, e uma pressão de trabalho que é efetuada pela contração muscular, promovem efeitos que incluem aumento da pressão tecidual e do fluxo venoso (Carati et al., 2010; Heinig et al., 2015).

No membro afetado pelo linfedema ocorrem alterações na hemodinâmica do sistema linfático, circulação arterial e venosa, explicado pela hipótese de Starling, e uma forma de controle é por meio da compressão do membro associado a contração musculoesquelética, que leva a um aumento da pressão intersticial, ajudando no controle e redução do edema (Dennis, 2008).

O membro acometido pelo linfedema secundário ao tratamento do câncer de mama quando comparado ao membro saudável é acometido por alterações circulatórias 
sanguíneas, arteriais e venosas, sendo o principal achado o incremento do fluxo no membro acometido, pela estenose de vasos (Matheus et al., 2011; Rezende et al., 2011; Valinote et al., 2013; Guirro et al., 2015). Além disso, um estudo de Fong et al. (2014) observou que após um protocolo de exercícios nos membros superiores de mulheres com linfedema houve incremento da circulação sanguínea arterial, pelo provável comprometimento cirúrgico da circulação linfática e venosa, estando de acordo com os achados do presente estudo.

Estudo de Rezende et al. (2017) observou que os efeitos da cinesioterapia combinada com a terapia compressiva promovem incremento da velocidade média do fluxo sanguíneo nas artérias e veias axilares e braquiais, em comparação ao grupo que recebeu apenas cinesioterapia, não encontrou diferenças significativas entre diferentes terapias compressivas (enfaixamento compressivo funcional e braçadeira elástica). Resultado semelhante aos encontrados no presente estudo que encontrou aumento do fluxo sanguíneo arterial e venoso após a movimentação do membro acometido aliado a ambos ECF.

Diferente da maioria dos estudos que envolvem avalição do linfedema decorrente do tratamento do câncer de mama, o foco do presente estudo não foi a redução do volume do membro. Os resultados da comparação da pressão exercida pelas diferentes técnicas de ECF (espiral e oito), no repouso e durante o movimento, apresentou diferença significativa apenas durante o movimento, em que a pressão exercida sobre o tecido com a técnica em oito foi maior quando comparado a técnica em espiral.

O efeito de diferentes técnicas de ECF na pressão exercida, influenciada por diferentes fatores com diferentes técnicas e aplicações avaliadas demonstrou que a técnica em espiral apresentou uma pressão menor durante o movimento quando comparado a técnica em oito. Resultado semelhante ao estudo de Oh et al., 2019, que também comparou as mesmas técnicas de ECF na redução do volume e na incapacidade do membro, e observaram diferença significativa com a técnica em oito na redução do 
volume, além de melhora na incapacidade do membro superior quando comparado a técnica em espiral.

O presente estudo observou um prejuízo no tempo de execução do teste de função manual de Jebsen Tayor em ambas técnicas de enfaixamento compressivo funcional, quando comparado sem enfaixamento. A utilização da compressão compromete a movimentação, entretanto, controla o edema, principal agente complicador da mobilidade do membro. Vários fatores podem influenciar neste aspecto, como a pressão exercida, bem como o número de faixas utilizadas.

O ideal é que se tenha um número de faixas que restrinja minimamente o movimento do membro, e consequentemente a circulação linfática e sanguínea, diretamente relacionadas ao controle do volume do membro. O presente estudo apresenta como limitação da identificação de qual o número ideal de faixas para o controle do linfedema, uma vez que a padronização foi necessária para comparação entre as técnicas. O presente estudo é pioneiro na avaliação da funcionalidade do membro acometido por linfedema por meio do teste de Jebsen Taylor, entretanto, encontra-se na literatura avaliações em outras populações, como distrofia muscular, distúrbios de membros superiores e na doença de Parkinson (Allgöwer et al., 2017; Opara et al., 2017; Artilheiro et al., 2018). A funcionalidade do membro com e sem ECF, apontou prejuízo na execução do teste quando efetuado com as diferentes técnicas de ECF (espiral e oito), sendo que o tempo de execução foi maior quando realizado com ambas técnicas. Entretanto, não houve diferença significativa na comparação entre ambas as técnicas estudadas.

O presente estudo encontrou uma reprodutibilidade e confiabilidade baixa a moderada interexaminador, e baixa a alta intraexaminador na aplicação do ECF, fato que confirma a hipótese do estudo, em que o ECF apresenta um método aplicador dependente.

A técnica do ECF pode apresentar diferenças em sua execução, sendo que cada profissional pode aplicar pressões distintas com as ataduras, além de diferenças individuais na execução das técnicas. Apesar da execução da técnica apresentar o caráter 
“aplicador-dependente", os resultados demonstram efeitos positivos na circulação, entretanto, novos estudos são necessários para desvendar a questão do número ideal de ataduras que permita pressão adequada, no entanto, sem comprometer a funcionalidade, promovendo consequentemente maior eficiência no tratamento e controle do linfedema decorrente do tratamento do câncer de mama. 
Banclusiño 


\section{CONCLUSÃO}

Diante dos resultados obtidos é possível concluir que as hipóteses foram confirmadas:

- O enfaixamento compressivo funcional em espiral com quatro camadas apresenta reprodutibilidade e confiabilidade baixa a alta interexaminador e intraexaminador;

- Ambas as técnicas produziram incremento da circulação sanguínea do membro afetado por linfedema;

- A técnica em oito apresentou maior pressão em movimento quando comparado a em espiral;

- Ambas as técnicas de ECF apontaram prejuízo na funcionalidade do membro. 
Oreferéncias 


\section{REFERÊNCIAS}

Allgower K, Hermsdörfer J. Fine motor skills predict performance in the Jebsen Taylor hand function test after stroke. Clin Neurophysiol. 2017; 128 (10): 1858-1871.

Andersen L, Højris I, Erlandsen M, Andersen J. Treatment of breast-cancer-related lymphedema with or without manual lymphatic drainage--a randomized study. Acta Oncol. 2000; 39(3): 399-405.

Artilheiro MC, Fávero FM, Caromano FA, Oliveira ASB, Junior NC, Voos MC, Cardoso de Sá CS. Reliability, validity and description of timed performance of the Jebsen-Taylor Test in patients with muscular dystrophies. Braz J Phys Ther. 2018; 22 (3): 190-197.

Baumann FT, Bloch W, Weissen A, Brockhaus M, Beulertz J, Zimmer P, Streckmann F, Zopf EM. Physical activity in breast cancer patients during medical treatment and in the aftercare - a review. Breast Care. 2013; 8(5): 330-334.

Beckman JA, Thakore A, Kalinowski BH, Harris JR, Creager MA. Radiation therapy impairs endothelium-dependent vasodilation in humans. J Am Coll Cardiol. 2001;37(3):761-5.

Brito GN, Brito LS, Paumgartten FJ, Lins MF. Lateral preferences in Brazilian adults: an analysis with the Edinburgh Inventory. Cortex. 1989; 25(3): 403-15.

Bunke N, Brown K, Bergan J. Phlebolymphemeda: usually unrecognized, often poorly treated. Perspect Vasc Surg Endovasc Ther 2009; 21 (2): 65-8.

Colin Carati, Bren Gannon, Neil Piller. Anatomy and physiology in relation to compression of the upper limb and thorax. Journal of Lymphoedema. 2010; 5(1): 58-67.

Culicchia G, Nobilia M, Asturi M, Santilli V, Paoloni M, Santis R, Galeoto G. Crosscultural adaptation and validation of the Jebsen-Taylor hand function test in an Italian population. Rehabil Res Pract. 2016; 2016, 8970917.

Damstra RJ, Partsch H. Compression therapy in breast cancer-related lymphedema: A randomized, controlled comparative study of relation between volume and interface pressure changes. J Vasc Surg. 2009; 49(5): 1256-63.

Dayes IS, Whelan TJ, Julian JA, Parpia S, Pritchard KI, D’Souza DP, Kligman L, Reise D, LeBlanc L, McNeely ML, Manchul L, Wiernikowski J, Levine MN. Randomized trial of decongestive lymphatic therapy for the treatment of lymphedema in women with breast cancer. J Clinil Oncol 2013;31 (30): 3758-63.

Dennis R. Haemodynamics of lymphoedemaJournal of Lymphoedema,2008;3(2):45-9.

Ergin G, Şahinoğlu E, Karadibak D, Yavuzşen T. Effect of bandage compliance on upper extremity volume in patients with breast cancer-related lymphedema. Lymphat Res Biol. 2018; 16 (6):553-558.

Ezzo J, Manheimer E, McNeely ML, Howell DM, Weiss R, Johansson KI, Bao T, Bily L, Tuppo CM, Williams AF, Karadibak D. Manual lymphatic drainage for lymphedema 
following breast cancer treatment. Cochrane Database Syst Rev. 2015; (5): CD003475.

Fleiss J. The design and analysis of clinical experiments. New York: Wiley;1986.

Fong SS, Ng SS, Luk WS, et al. Effects of qigong exercise on upper limb lymphedema and blood flow in survivors of breast cancer: a pilot study. Integr Cancer Ther. 2014;13(1):54-61.

Franzeck UK, Spiegel I, Fischer M, et al. Combined physical therapy for lymphedema evaluated by fluorescence microlymphography and lymph capillary pressure measurements. J Vasc Res 1997;34 (4): 306-11.

Fu MR, Deng J, Armer JM. Putting evidence into practice: cancer-related lymphedema. Clin J Oncol Nurs. 2014;3(18):68-79.

Fu MR, Ridner SH, Hu SH, Stewart BR, Cormier JN, Armer JM. Psychosocial impact of lymphedema: a systematic review of literature from 2004 to 2011. Psychooncology. 2013; 22(7): 1466-84.

Gärtner R, Jensen MB, Kronborg L, Ewertz M, Kehlet H, Kroman N. Self-reported armlymphedema and functional impairment after breast cancer treatment - A nationwide study of prevalence and associated factors. Breast. 2010; 19(6):506-15.

Grada AA, Phillips TJ. Lymphedema: Pathophysiology and Clinical Manifestations. J Am Acad Dermatol. 2017; 77(6):1009-1020.

Guirro EC, Guirro RR, Dibai-Filho AV, Pascote SC, Rodrigues-Bigaton D. Immediate effects of electrical stimulation, diathermy, and physical exercise on lower limb arterial blood flow in diabetic women with peripheral arterial disease: a randomized crossover trial. J Manipulative Physiol Ther. 2015;38(3):195-202.

Heinig B, Wollina U. [Complex decongestive therapy]. Hautarzt. 2015; 66(11): 810-8.

Johansson K, Piller N. Weight-bearing exercise and its impact on arm lymphoedema. J Lymphoedema. 2007;2(1):15-22.

Johansson K, Tibe K, Weibull A, Newton RC. Low intensity resistance exercise for breast cancer patients with arm lymphedema with or without compression sleeve. Lymphology. 2005; 38(4): 167-80.

Johnson KC, DeSarno M, Ashikaga T, Dee J, Henry SM. Ultrasound and clinical measures for lymphedema. Lymphat Res Biol. 2016;14(1):8-17.

Kang Y, Jang DH, Jeon JY, Lee SJ, Jeong SY, Shin DI, Kim HJ. Pressure monitoring of multilayer inelastic bandaging and the effect of padding in breast cancer-related lymphedema patients. Am J Phys Med Rehabil. 2012; 91(9): 768-73.

Karadibak D, Yavuzsen T. Evaluation of kinesthetic sense and hand function in women with breast cancer-related lymphedema. J Phys Ther Sci. 2015; 27(6): 1671-1675.

Karakashian K, Pike C, Loon RV. Computational Investigation of the Laplace Law in compression therapy. J Biomech. 2019; 85:6-17.

King M, Deveaux A, White H, Rayson D. Compression garments versus compression 
bandaging in decongestive lymphatic therapy for breast cancer-related lymphedema: a randomized controlled trial. Support Care Cancer. 2012; 20 (5): 1031-6.

Kwan ML, Darbinian J, Schmitz KH, Citron R, Partee P, Kutner SE, Kushi LH. Risk factors for lymphedema in a prospective breast cancer survivorship study: the Pathways Study. Arch Surg. 2010; 145(11):1055-63.

Lamprou DA, Damstra RJ, Partsch H. Prospective, randomized, controlled trial comparing a new two-component compression system with inelastic multicomponent compression bandages in the treatment of leg lymphedema. Dermatol Surg. 2011; 37(7): 985-91.

Lasinski BB, McKillip Thrift K, Squire D, Austin MK, Smith KM, Wanchai A, Green JM, Stewart BR, Cormier JN, Armer JM. A systematic review of the evidence for complete decongestive therapy in the treatment of lymphedema from 2004 to 2011. PM R. 2012; 4(8): 580-601.

Lazareth I. Classification of lymphedema. Rev Med Interne. Suppl. 2002; 3: 375-8.

Lima KCA, Francisco MM, Freitas PB. Relationship among performance in different tests often used for hand function assessment. Fisioter Mov. 2012; 25(3): 517-24.

Lima KCA, Freitas PB. Hand function and power grip strength assessment in individuals with diabetes mellitus. Fisioter. Pesqui. 2012; 19(4): 375-380.

The Diagnosis and Treatment of Peripheral Lymphedema: 2016 Consensus Document of the International Society of Lymphology. Lymphology. 2016 Dec; 49(4): 170-84.

Matheus, CN; Guirro, ECO. Change in blood flow velocity demonstrated by Doppler ultrasound in upper limb after axillary dissection surgery for the treatment of breast cancer. Breast Cancer Res Treat. 2011; 12(7): 697-704.

Mayrovitz HN. The standard of care for lymphedema: current concepts and physiological considerations. Lymphat Res Biol 2009; 7(2): 101-8.

McLaughlin SA, Wright MJ, Morris KT, Giron GL, Sampson MR, Brockway JP, Hurley KE, Riedel ER, Van Zee KJ. Prevalence of lymphedema in women with breast cancer 5 years after sentinel lymph node biopsy or axillary dissection: objective measurements. $\mathbf{J}$ Clin Oncol. 2008; 26(32): 5213-9.

Mondry TE, Riffenburgh RH, Johnstone PA. Prospective trial of complete decongestive therapy for upper extremity lymphedema after breast cancer therapy. Cancer J. 2004; 10(1): 42-8.

Moseley AL, Carati CJ, Piller NB. A systematic review of common conservative therapies for arm lymphoedema secondary to breast cancer treatment. Ann Oncol. 2007; 18(4): 63946.

Munro BH. Correlation. In: Munro BH. Statistical methods for health care research. 4a ed. Philadelphia: Lippincott; 2001. p. 223-43.

Nascimben Matheus C. Guirro ECO. Change in blood flow velocity demonstrated by Doppler ultrasound in upper limb after axillary dissection surgery for the treatment of breast cancer. Breast Cancer Res Treat 2011; 127:697-704. 
consensus for the management of peripheral arterial disease (TASC II). J Vasc Surg. 2007;45 Suppl S:S5-67.

Oh SH, Ryu SH, Jeong HJ, Lee JH, Sim YJ. Effects of different bandaging methods for treating patients with breast cancer-related lymphedema. Ann Rehabil Med. 2019 Dec;43(6):677-685.

Olszewski WL. Pathophysiological aspects of lymphedema of human limbs: I. Lymph protein composition. Lymph Res Biol 2003;1 (3):235-43.

Opara J, Malecki A, Malecka E, Socha T. Motor assessment in parkinson`s disease. Ann Agric Environ Med. 2017; 24 (3): 411-415.

Partsch H. (2011) Prospective, randomized, controlled trial comparing a new twocomponent compression system with inelastic multicomponent compression bandages in the treatment of leg lymphedema. Dermatol Surg. 37 (7): 985-91.

Partsch H, Clark M, Mosti G, Steinlechner E, Schuren J, Abel M, Benigni JP, ColeridgeSmith P, Cornu-Thénard A, Flour M, Hutchinson J, Gamble J, Issberner K, Juenger M, Moffatt C, Neumann HAM, Rabe E, Uhl JF, Zimmet S. Classification of compression bandages: Practical Aspects. Dermatol Surg. 2008; 34 (5): 600-9.

Partsch H. Compression therapy: clinical and experimental evidence. Ann Vasc Dis. 2012;5(4):416-422.

Partsch H, Damstra RJ, Mosti G. Dose finding for an optimal compression pressure to reduce chronic edema of the extremities. Int Angiol. 2011; 30(6): 527-33.

Partsch H, Stout N, Forner-Cordero I, Flour M, Moffatt C, Szuba A, Milic D, Szolnoky G, Brorson H, Abel M, Schuren J, Schingale F, Vignes S, Piller N, Döller W. Clinical trials needed to evaluate compression therapy in breast cancer related lymphedema (BCRL). Proposals from an expert group. Int Angiol. 2010; 29(5): 442-53.

Paskett ED, Dean JA, Oliveri JM, Harrop JP. Cancer-related lymphedema risk factors, diagnosis, treatment, and impact: a review. J Clin Oncol. 2012; 30(30): 3726-33.

Pellicer J, Garcia-Morales V, Hernandez M. On the demonstration of the Young-Laplace equation in introductory physics courses. Phys. Educ. 2000; 35 (2): 126-129.

Petrek JA, Pressman PI, Smith RA. Lymphedema: current issues in research and management. CA Cancer J Clin. 2000;50(5):292-307

Portney LG, Watkins MP. Reliability. In: Portney LG, Watkins MP, editores. Foundations of clinical research. 2a ed. New Jersey: Prentice Hall Health; 2000. P. 61-75.

Randheer S, Kadambari D, Srinivasan K, Bhuvaneswari V, Bhanumathy M, Salaja R. Comprehensive decongestive therapy in postmastectomy lymphedema: An Indian perspective. Indian J Cancer .2011; 480(4): 397-402.

Rezende LF. Evaluation of lymphatic compensation by lymphoscintigraphy in the postoperative period of breast cancer surgery with axillary dissection. Tumori. 2011;97(3):309-15.

Rezende MS, Marsengo AL, Apolinário A, Ferreira VTK, Guirro ECO. Correlation 
between upper limb volume and arterial and venous blood flow velocity in lymphedema secondary to breast cancer treatment. J Manipulative Physiol Ther. 2017; 40(4):241-245.

Rezende MS, Marsengo AL, de Jesus Guirro RR, Guirro ECO. Blood flow velocity in brachial and subclavian vessels immediately after compressive procedures for treatment of postcancer therapy lymphedema in breast cancer: a randomized blind clinical trial. Lymphat Res Biol. 2017; 15(1):23-31.

Sander AP. Upper-extremity volume measurements in women with lymphedema: A comparison of measurements obtained via water displacement with geometrically determined volume. Phys Ther. 2002; 8 (2) 1201-1212.

Szuba A, Cooke JP, Yousuf S, Rockson SG. Decongestive lymphatic therapy for patients with cancer-related or primary lymphedema. Am J Med. 2000; 109(4): 296-300.

Tambour M, Tange B, Christensen R, Gram B. Effect of physical therapy on breast cancer related lymphedema: protocol for a multicenter, randomized, single-blind, and equivalence trial. BMC Cancer. 2014; 34(14): 239-43. 20.

Terwee CB, Bot SD, de Boer MR, van der Windt DA, Knol DL, Dekker J, et al. Quality criteria were proposed for measurement properties of health status questionnaires. J Clin Epidemiol. 2007; 60: 34-42.

Valinote SP, Freitas-Junior R, Martins KA, Pereira AC, Pereira CE, Martins E. Alterações venosas e linfáticas em mulheres com linfedema após linfadenectomia axilar no tratamento do câncer de mama. Rev Bras Ginecol Obstet. 2013;35(4):171-7.

Vignes S. Lymphedema: From diagnosis to treatment. Rev Med Interne. 2017; 38(2): $97-$ 105.

Vignes S, Porcher R, Arrault M, Dupuy A. Long-term management of breast cancerrelated lymphedema after intensive decongestive physiotherapy. Breast Cancer Res Treat. 2007; 101(3): 285-90.

Weir JP. Quantifying test-retest reliability using the intraclass correlation coefficient and the SEM. J Strength Cond Res. 2005; 19:231-40. 
ANEXO A

USP - HOSPITAL DAS
CLINICAS DA FACULDADE DE
MEDICINA DE RIBEIRÃO
PARECER CONSUBSTANCIADO DO CEP

DADOS DO PROJETO DE PESQUISA

Título da Pesquisa: CONFIABILIDADE, REPRODUTIBILIDADE E EFICIËNCIA DO ENFAIXAMENTO COMPRESSIVO FUNCIONAL, NO TRATAMENTO DO LINFEDEMA SECUNDARIO AO TRATAMENTO DO CANCER DE MAMA

Pesquisador: Elaine Caldeira de Oliveira Guimo

Área Temática:

Versão: 2

CAAE: 90238218.7 .0000 .5440

Instituiçäo Proponente:UNIVERSIDADE DE SAO PAULO

Patrocinador Principal: Financiamento Próprio

\section{DADOS DO PARECER}

Número do Parecer: 2.748 .559

Apresentaçäo do Projeto:

Os autores se propöem a estuclar a confiabilidade e a reprodutbilidade de duas técnicas de enfaixamento compressivo funcional (ECF) em 50 pacientes, com 40 a 70 anos de idade, com linfedema decorrente da cirurgia para extippar căncer de mama. As participantes são acompanhadas em ambulatório do Núcleo de Ensino Pesquisa e Reabilitaçäo de Mastectomizadas (REMA), da Escola de Enfermagem de Ribeirão Preto (EERPIUSP).

O estudo está dividido em dois subprojetos: o subprojeto 1 consiste em um estudo de confiabilidade $e$ reprodutibilidade, e o subprojeto 2 consiste em estudo observacional randomizado cego tipo crossover.

Objetivo da Pesquisa:

Avaliar a confiabilidade intra e interexaminador da técnica de enfaixamento compressivo, e a funcionalidade do membro bem como a influência na circulaçăo sanguinea de diferentes técnicas de enfaixamento compressivo funcional.

Objetivos especificos:

Avaliar a confiabilidade intraexaminador e interexaminador na pressäo exercida pela técnica em espiral do enfaixamento compressivo com quatro camadas;

- Avaliar a eficiència de duas técnicas de enfaixamento compressivo com quatro camadas na pressão exercida por um avaliador:

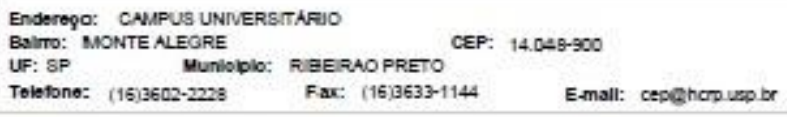




USP - HOSPITAL DAS
CLINICAS DA FACULDADE DE
MEDICINA DE RIBEIRÃO Protoformo

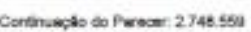

- Avaliar a eficiéncia de duas técnicas de enfaixamento compressivo com quatro camadas no teste de Jebsen Taylor na funcionalidade do membro superior;

- Avaliar o efeito da circulaçäo sanguínea antes e após no teste de Jebsen Taylor na funcionalidade do membro superior nas duas técnicas de enfaixamento compressivo.

Avaliaçäo dos Riscos e Beneficios:

Realizada adequadamente.

Comentários e Considerações sobre a Pesquisa:

O projeto em questäo contém méritos, pois pretende avaliar os desfechos do enfaixamento compressivo funcional de membro superior de pacientes com linfedema secundário à cirurgia de exérese do tumor.

Consideraçöes sobre os Termos de apresentação obrigatória:

Os termos de apresentaçäo obrigatória estäo adequados.

As correçöes sugeridas foram contempladas na nova versão, especialmente aquelas refentes ao TCLE

Conclusöes ou Pendências e Lista de Inadequaçöes:

Diante do exposto e à luz da Resolução CNS 466/2012, o projeto de pesquisa, assim como o Termo de Consentimento Livre e Esclarecido Versäo 2 - 08/06/2018, podem ser enquadrados na categoria APROVADO.

Consideraçöes Finais a critério do CEP:

Projeto Aprovado: Tendo en vista a legislação vigente, deven ser encaminhados ao CEP, relatórios parciais anuais referentes ao andamento da pesquisa e relatório final ao término do trabalho. Qualquer modificaçăo do projeto original deve ser apresentada a este CEP em nova versäo, de forma objetiva e com justificativas, para nova apreciação

Este parecer foi elaborado baseado nos documentos abaixo relacionados:

\begin{tabular}{|c|c|c|c|c|}
\hline Tipo Documento & Arquivo & Postagem & Autor & Stuaçä́o \\
\hline $\begin{array}{l}\text { Informaçóes Básicas } \\
\text { do Projeto }\end{array}$ & $\begin{array}{l}\text { PB_INFORMACOES_BASICAS_DO_P } \\
\text { ROJETO } 1131134 . \mathrm{pot}\end{array}$ & $\begin{array}{c}08 / 06 / 2018 \\
16: 07: 27\end{array}$ & & Aceito \\
\hline Outros & CORRECOES_TCLE.pd & $\begin{array}{l}08 / 06 / 2018 \\
16: 06: 49\end{array}$ & $\begin{array}{l}\text { Elaine Caldeira de } \\
\text { Oliveira Guimo }\end{array}$ & Aceito \\
\hline $\begin{array}{l}\text { TCLE / Termos de } \\
\text { Assentimento I } \\
\text { Justificativa de }\end{array}$ & $\begin{array}{l}\text { Terno_consentimento_2_08_06_18_nov } \\
\text { aversaopdf }\end{array}$ & $\begin{array}{l}0800 / 2018 \\
16: 04: 43\end{array}$ & $\begin{array}{l}\text { Elaine Caldeira de } \\
\text { Oliveira Guimo }\end{array}$ & Aceito \\
\hline
\end{tabular}

Enderop̧O: CAMPUS UNIVERSTARIO

BolTO: MONTE ALEGRE

CEP: $14.040-900$

Toletone: (16)3602-2228 Fax: (16)3633-1144 E-mall: cepGhopuep br 


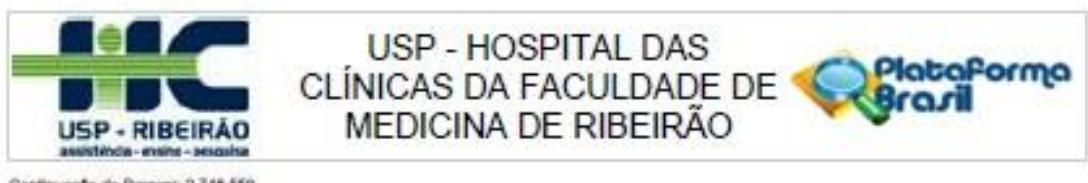

\begin{tabular}{|c|c|c|c|c|}
\hline Auséncia & $\begin{array}{l}\text { Terno_consentimento_2_08_06_18_nov } \\
\text { aversaio ooff }\end{array}$ & $\begin{array}{l}0806 / 2018 \\
16: 04: 43\end{array}$ & $\begin{array}{l}\text { Elaine Caldeira de } \\
\text { Oliveira Guimo }\end{array}$ & Aceito \\
\hline $\begin{array}{l}\text { TCLE / Termos de } \\
\text { Assentimento / } \\
\text { Justificativa de } \\
\text { Auséncia }\end{array}$ & $\begin{array}{l}\text { Termo_consentimento_1_08_06_18_no } \\
\text { vaversao.poff }\end{array}$ & $\begin{array}{l}08 / 06 / 2018 \\
16: 04: 25\end{array}$ & $\begin{array}{l}\text { Elaine Caldeira de } \\
\text { Oliveira Guimo }\end{array}$ & Aceito \\
\hline Orçamento & img005.pdf & $\begin{array}{l}05 / 06 / 2018 \\
15: 36: 39\end{array}$ & $\begin{array}{l}\text { Elaine Caldeira de } \\
\text { Oliveira Guimo }\end{array}$ & Aceito \\
\hline $\begin{array}{l}\text { Projeto Detalhado / } \\
\text { Brochura } \\
\text { Investigador }\end{array}$ & Projeto.pdf & $\begin{array}{l}2305 / 2018 \\
20: 55: 23\end{array}$ & $\begin{array}{l}\text { Elaine Caldeira de } \\
\text { Oliveira Guimo }\end{array}$ & Aceito \\
\hline Folha de Rosto & Folha_de_rostopdf & $\begin{array}{l}08 / 05 / 2018 \\
16: 09: 13\end{array}$ & $\begin{array}{l}\text { Elaine Caldeira de } \\
\text { Oliveira Guimo }\end{array}$ & Aceito \\
\hline $\begin{array}{l}\text { TCLE / Termos de } \\
\text { Assentimento / } \\
\text { Justificativa de } \\
\text { Ausência }\end{array}$ & Terno_consentimento_2.pdf & $\begin{array}{c}0805 / 2018 \\
09: 40: 39\end{array}$ & $\begin{array}{l}\text { Elane Caldera de } \\
\text { Oliveira Guimo }\end{array}$ & Aceto \\
\hline $\begin{array}{l}\text { TCLE / Termos de } \\
\text { Assentimento / } \\
\text { Justificativa de } \\
\text { Ausência }\end{array}$ & Termo_consentimento_1.poff & $\begin{array}{c}00 / 05 / 2018 \\
00: 40: 25\end{array}$ & $\begin{array}{l}\text { Elaine Caldeira de } \\
\text { Oliveira Guimo }\end{array}$ & Aceito \\
\hline $\begin{array}{l}\text { Dedaraçáo de } \\
\text { Instituiçáo e } \\
\text { Infraestuutura }\end{array}$ & Comite_de_etica.pdf & $\begin{array}{c}08 / 05 / 2018 \\
15: 42: 31\end{array}$ & $\begin{array}{l}\text { Elaine Caldeira de } \\
\text { Oliveira Guimo }\end{array}$ & Aceito \\
\hline
\end{tabular}

Situagão do Parecer:

Aprovado

Necessita Apreciaçäo da CONEP:

Nă้

RIBEIRAO PRETO, 02 de Julho de 2018

Assinado por:

MARCLA GUIMARÄES VILANOVA

(Coordenador)

EnderogO: CNMPUS UNIVERSTARIO

Bolmo: MONTE ALEGRE

UF: $S P$ CEP: 14.049-900

Tolefone: (16)3602-2228 Fax: (16)3633-1144 E-mall: cepehcrouspor 


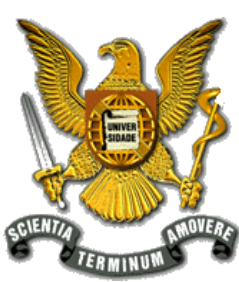

\title{
APÊNDICE A \\ UNIVERSIDADE DE SÃO PAULO FACULDADE DE MEDICINA DE RIBEIRÃO PRETO
}

\author{
TERMO DE CONSENTIMENTO LIVRE E ESCLARECIDO
}

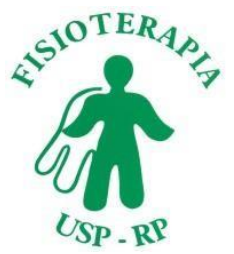

Você está sendo convidada a participar como voluntária da primeira etapa do projeto de pesquisa "Confiabilidade, reprodutibilidade e eficiência do enfaixamento compressivo funcional, no tratamento do linfedema secundário ao tratamento do câncer de mama", coordenado pela Profa. Dra. Elaine C. de O. Guirro e desenvolvido pela aluna Amanda Apolinário, no qual serão recrutadas 50 voluntárias, que tem por objetivo avaliar se a técnica do enfaixamento compressivo funcional em espiral é confiável, e se existe diferença na realização da técnica entre os profissionais. Este projeto tem como finalidade ajudar a compreensão da melhor forma de tratamento do linfedema (edema ou inchaço).

Se você aceitar participar do estudo, será agendado um horário e data combinado entre você e o pesquisador para o início das atividades. Você deverá comparecer ao REMA (Núcleo de Pesquisa e Ensino de Mulheres Mastectomizadas), situado na Escola de Enfermagem, para a realização da entrevista (preenchimento dos dados pessoais e avaliação física individual). Serão realizadas duas intervenções com duração de aproximadamente 1 hora cada, uma vez na semana, durante duas semanas seguidas. A sessão será iniciada: com o primeiro avaliador que irá realizar a técnica de enfaixamento com quatro camadas de ataduras (faixas) em espiral, em seguida o segundo avaliador realizará o mesmo procedimento; análise da pressão exercida pelo enfaixamento por dois avaliadores diferentes; após 7 dias irá realizar o mesmo procedimento. O equipamento utilizado para medir a pressão do enfaixamento, é semelhante ao equipamento utilizado para medira a pressão arterial, porém sem pressionar o membro.

Os riscos são mínimos, uma vez que envolve exames executados rotineiramente, podendo causar certo desconforto pela maior duração dos exames, pela necessidade de repetição, mas nos comprometemos a realizá-las com o menor desconforto possível, e são exames que já são efetuados rotineiramente. Asseguramos que seu nome e identidade serão mantidos em sigilo. Os dados coletados serão utilizados apenas nesta pesquisa e ficarão com a orientadora do projeto em arquivo do laboratório, cujo acesso é restrito aos pesquisadores. O pesquisador também tem o direito de excluir seus dados deste estudo caso não participe de todas as avaliações ou pela não adequação ao objetivo desse trabalho. Ao final os resultados serão divulgados em artigos e eventos científicos. Você tem o direito ao acesso dos resultados da pesquisa. Sua participação é voluntária, isto é, não há pagamento por sua colaboração. Todas as despesas que você tiver relacionadas diretamente ao projeto de pesquisa, tais como transporte e alimentação serão cobertas pelo pesquisador responsável. Caso haja algum dano direto ou indireto decorrente de sua participação na pesquisa, você poderá ser indenizado, obedecendo-se as conforme as leis vigentes do país.

É seu direito ter uma via deste documento assinado pelo(a) Senhor(a) e pela pesquisadora, e no caso de desistência ou recusar-se a participar, o fato não ocasionará penalidade ou prejuízo a sua pessoa, bem como ao atendimento efetuado no REMA. Você receberá resposta a qualquer pergunta ou esclarecimento acerca dos procedimentos, riscos, benefícios, outras questões relacionadas com a pesquisa.

O Comitê de Ética em Pesquisa também tem a finalidade de proteger as pessoas que participam da pesquisa e preservar seus direitos. Assim, se for necessário, entre em contato com o CEP do HCFMRP/USP pelo telefone (16) 3602-2228 ou pelo seu endereço - Campus Universitário, Monte Alegre - Ribeirão Preto - SP, no horário das 8 às $17 \mathrm{~h}$.

Caso você tenha compreendido o que lhe foi exposto e esteja de acordo em participar deste projeto, favor assinar e rubricar as duas vias do TCLE, uma das quais lhe pertence e a outra ficará sob a guarda da equipe de pesquisa.

Data:

Nome da Voluntária

Assinatura da Voluntária
Data:

Nome da Pesquisadora Responsável

Para maiores informações e esclarecimentos, entre em contato com a orientadora, Profa. Dra. Elaine Caldeira de Oliveira Guirro (Tel: 3602-4584), ou com a pesquisadora responsável, Ft. Amanda Apolinário (Cel: (19) 99222-5480) 


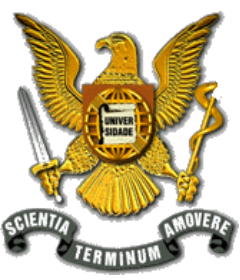

\section{UNIVERSIDADE DE SÃO PAULO FACULDADE DE MEDICINA DE RIBEIRÃO PRETO}

\section{TERMO DE CONSENTIMENTO LIVRE E ESCLARECIDO}

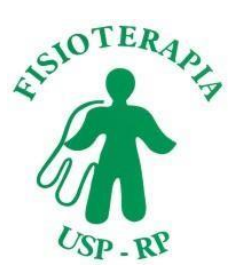

Você está sendo convidada a participar como voluntária da segunda etapa do projeto de pesquisa "Confiabilidade, reprodutibilidade e eficiência do enfaixamento compressivo funcional, no tratamento do linfedema secundário ao tratamento do câncer de mama”, coordenado pela Profa. Dra. Elaine C. de O. Guirro e desenvolvido pela aluna Amanda Apolinário, em que das 50 voluntárias da primeira etapa 25 destas serão recrutadas a participar da segunda etapa, que tem por objetivo verificar a eficiência de diferentes técnicas de enfaixamento compressivo funcional na funcionalidade e circulação do membro superior afetado por linfedema. Este projeto tem como finalidade ajudar a compreensão da melhor forma de tratamento do linfedema (inchaço do membro superior).

Se você aceitar participar do estudo, será agendado um horário e data combinado entre você e o pesquisador para o início das atividades. Você deverá comparecer ao REMA (Núcleo de Pesquisa e Ensino de Mulheres Mastectomizadas), situado na Escola de Enfermagem, para a realização da entrevista (preenchimento dos dados pessoais e avaliação física individual). Será realizada uma intervenção com duração de aproximadamente 2 horas, uma vez na semana, durante duas semanas seguidas. A sessão será sorteada de acordo com a técnica que deverá ser iniciada: um dia será realizado a técnica de enfaixamento compressivo funcional em espiral ou em oito, na semana seguinte muda o tipo de enfaixamento. Será analisada a pressão exercida pelo enfaixamento compressivo através de um equipamento semelhante ao utilizado para medira a pressão arterial, porém sem pressionar o membro, a avaliação da funcionalidade do membro superior pelo teste que se assemelha as atividade de vida diária, e será analisada a pressão arterial de membros superiores e inferiores (braços e pernas), com equipamento de medir pressão digital, sendo os valores utilizados em cálculo para avaliar a condição da circulação arterial. Também será avaliada a circulação das artérias dos braços com equipamento Ultrassom Doppler, que toca a pele untada com gel; após 7 dias irá realizar o mesmo procedimento.

Os riscos são mínimos, uma vez que envolve exames executados rotineiramente, podendo causar certo desconforto pela maior duração dos exames, pela necessidade de repetição, mas nos comprometemos a realizá-las com o menor desconforto possível. Você pode ser beneficiada diretamente com sua participação, pois receberá informações dos resultados da pesquisa, sobre qual técnica do enfaixamento compressivo funcional é mais eficaz.

Asseguramos que seu nome e identidade serão mantidos em sigilo. Os dados coletados serão utilizados apenas nesta pesquisa e ficarão com a orientadora do projeto em arquivo do laboratório, cujo acesso é restrito aos pesquisadores. O pesquisador também tem o direito de excluir seus dados deste estudo caso não participe de todas as avaliações ou pela não adequação ao objetivo desse trabalho. Ao final os resultados serão divulgados em artigos e eventos científicos. Você tem o direito ao acesso dos resultados da pesquisa. Sua participação é voluntária, isto é, não há pagamento por sua colaboração. Todas as despesas que você tiver relacionadas diretamente ao projeto de pesquisa, tais como transporte e alimentação serão cobertas pelo pesquisador responsável. Caso haja algum dano direto ou indireto decorrente de sua participação na pesquisa, você poderá ser indenizado, obedecendo-se as conforme as leis vigentes do país.

É seu direito ter uma via deste documento assinado pela Senhor(a) e pela pesquisadora, e no caso de desistência ou recusarse a participar, o fato não ocasionará penalidade ou prejuízo a sua pessoa, bem como ao atendimento efetuado no REMA. Você receberá resposta a qualquer pergunta ou esclarecimento acerca dos procedimentos, riscos, benefícios, outras questões relacionadas com a pesquisa.

O Comitê de Ética em Pesquisa também tem a finalidade de proteger as pessoas que participam da pesquisa e preservar seus direitos. Assim, se for necessário, entre em contato com o CEP do HCFMRP/USP pelo telefone (16) 3602-2228 ou pelo seu endereço - Campus Universitário, Monte Alegre - Ribeirão Preto - SP, no horário das 8 às $17 \mathrm{~h}$.

Caso você tenha compreendido o que lhe foi exposto e esteja de acordo em participar deste projeto, favor assinar e rubricar as duas vias do TCLE, uma das quais lhe pertence e a outra ficará sob a guarda da equipe de pesquisa.

Data:

Nome/Assinatura da Voluntária
Data:

Nome/Assinatura da Pesquisadora Responsável

Para maiores informações e esclarecimentos, entre em contato com a orientadora, Profa. Dra. Elaine Caldeira de Oliveira Guirro (Tel: 3602-4584), ou com a pesquisadora responsável, Ft. Amanda Apolinário (Cel: (19) 99222-5480) 